This is the author's final, peer-reviewed manuscript as accepted for publication. The publisher-formatted version may be available through the publisher's web site or your institution's library.

\title{
Semianalytical solution for dual-probe heat-pulse applications that accounts for probe radius and heat capacity
}

John H. Knight, Gerard J. Kluitenberg, Tamir Kamai, and Jan W. Hopmans

\section{How to cite this manuscript}

If you make reference to this version of the manuscript, use the following information:

Knight, J. H., Kluitenberg, G. J., Kamai, T., \& Hopmans, J. W. (2012). Semianalytical solution for dual-probe heat-pulse applications that accounts for probe radius and heat capacity. Retrieved from http://krex.ksu.edu

\section{Published Version Information}

Citation: Knight, J. H., Kluitenberg, G. J., Kamai, T., \& Hopmans, J. W. (2012). Semianalytical solution for dual-probe heat-pulse applications that accounts for probe radius and heat capacity. Vadose Zone Journal, 11(2).

Copyright: @ Soil Science Society of America

Digital Object Identifier (DOI): doi:10.2136/vzj2011.0112

Publisher's Link: https://www.soils.org/publications/vzj/articles/11/2/vzj2011.0112

This item was retrieved from the K-State Research Exchange (K-REx), the institutional repository of Kansas State University. K-REx is available at http://krex.ksu.edu 


\section{Semianalytical Solution for Dual-Probe Heat-Pulse Applications} that Accounts for Probe Radius and Heat Capacity

19 J. H. Knight, Faculty of Science and Technology, Queensland University of Technology,

20 Brisbane, QLD 4001, Australia; G. J. Kluitenberg, Department of Agronomy, Kansas State

21 University, Manhattan, KS 66506; and T. Kamai and J. W. Hopmans, Department of Land, Air

22 and Water Resources, University of California, Davis, CA 95616. Contribution no. 12-003-J

23 from the Kansas Agric. Exp. Stn., Manhattan, KS. *Corresponding author (gjk@ksu.edu). 


\section{Semianalytical Solution for Dual-Probe Heat-Pulse Applications \\ that Accounts for Probe Radius and Heat Capacity}

ABSTRACT

The dual-probe heat-pulse (DPHP) method is useful for measuring soil thermal properties.

Measurements are made with a sensor that has two parallel cylindrical probes: one for

introducing a pulse of heat into the soil (heater probe) and one for measuring change in

temperature (temperature probe). We present a semianalytical solution that accounts for the finite

radius and finite heat capacity of the heater and temperature probes. A closed-form expression

for the Laplace transform of the solution is obtained by considering the probes to be cylindrical

perfect conductors. The Laplace-domain solution is inverted numerically. For the case where

both probes have the same radius and heat capacity, we show that their finite properties have

equal influence on the heat-pulse signal received by the temperature probe. The finite radius of

the probes causes the heat-pulse signal to arrive earlier in time. This time-shift increases in

magnitude as probe radius increases. The effect of the finite heat capacity of the probes depends

on the ratio of the heat capacity of the probes $\left(C_{0}\right)$ and the heat capacity of the soil $(C)$.

Compared to the case where $C_{0} / C=1$, the magnitude of the heat-pulse signal decreases (i.e., smaller change in temperature) and the maximum temperature rise occurs later when $C_{0} / C>1$.

When $C_{0} / C<1$, the magnitude of the signal increases and the maximum temperature rise occurs earlier. The semianalytical solution is appropriate for use in DPHP applications where the ratio of probe radius $\left(a_{0}\right)$ and probe spacing $(L)$ satisfies the condition that $a_{0} / L \leq 0.11$. 


\section{INTRODUCTION}

2 The dual-probe heat-pulse (DPHP) method is widely used for measuring soil thermal

3 properties and volumetric water content. Measurements are made with a sensor that consists of

4 two parallel cylindrical probes. The heater probe contains electrical resistance wire used to

5 introduce heat at constant rate during a finite time interval (typically 8-12 seconds), and the

6 temperature probe contains a thermistor or thermocouple to measure change in temperature as a

7 function of time. If the distance between the probes and the rate and duration of heating are

8 known, soil thermal properties (i.e., volumetric heat capacity, thermal conductivity, and thermal

9 diffusivity) can be estimated from the transient temperature response by using an appropriate

10 solution of the heat conduction equation. Volumetric water content can be determined from the

11 volumetric heat capacity if the soil bulk density and the specific heat of the soil solid constituents

12 are known.

13 The solutions currently used in conjunction with the DPHP method treat the heater probe as

14 an infinite line source from which heat is released instantaneously (Campbell et al., 1991) or

15 during a finite time interval (Bristow et al., 1994). These solutions are generally regarded as

16 appropriate for determining thermal properties and water content, but they do not account for the

17 finite radius and finite thermal properties of the probes. By using numerical models, Guaraglia

18 and Pousa (1999) and Hopmans et al. (2002) demonstrated that thermal property estimates may

19 exhibit significant bias if the finite radius and finite thermal properties of the heater probe are not

20 taken into account. A growing body of experimental evidence also suggests that the DPHP

21 method may result in biased estimates of thermal properties and water content (Tarara and Ham,

22 1997; Song et al., 1998; Bristow et al., 2001; Basinger et. al., 2003; Ochsner et al., 2003; Ham

23 and Benson, 2004). To our knowledge, no attempt has been made to investigate the effects of the 
1 finite radius and finite thermal properties of the temperature probe. There is also need for a more

2 complete treatment of the effects caused by the finite properties of the heater probe. Quantifying

3 these effects is particularly relevant in light of the shift toward sensors with probes of larger

4 radius - a shift driven primarily by the need to make the sensor more robust (i.e., to minimize

5 deflection of the probes when inserted into soil). Campbell et al. (1991) used a sensor with a

6 probe radius of $0.407 \mathrm{~mm}$. In later work, sensors with a probe radius of $0.635 \mathrm{~mm}$ have been

7 used extensively (e.g., Tarara and Ham, 1997; Ren et al., 1999; Basinger et al., 2003), and results

8 have been reported for sensors with a probe radius as large as $1.0 \mathrm{~mm}$ (Nusier and Abu-Hamdeh, 9 2003).

10 We present a semianalytical solution that accounts for the finite radius and finite heat

11 capacity of the probes of a DPHP sensor. A closed-form expression for the Laplace transform of

12 the solution is obtained by considering the probes to be cylindrical perfect conductors of infinite

13 length. The algorithm of Stehfest (1970a,b) is used to invert the Laplace-domain solution

14 numerically. Precedent for use of the perfect conductor assumption can be found in the work of

15 Blackwell (1954) and Jaeger (1956), who used it to derive the solution for radial conduction

16 from a single heated probe in contact with a medium of infinite extent. This assumption seems

17 reasonable in the context of the DPHP method insofar as the probes of DPHP sensors typically

18 have a thermal conductivity much greater than that of soil. The solution presented herein is

19 similar to solutions in the groundwater literature that take into account the finite radius and finite

20 storage capacity of paired pumping and observation wells (Tongpenyai and Raghavan, 1981;

21 Ogbe and Brigham, 1984; Novakowski, 1989), but it avoids the need for numerical evaluation of

22 integrals in the Laplace domain. 
We begin by deriving a general form of the semianalytical solution, and then present a

2 special case of the solution useful for DPHP applications. The special case of the solution is used

3 to examine the effects of the finite properties of the probes. Specifically, we present results that

4 show how the heat-pulse signal received by the temperature probe is modified by the finite

5 radius and the finite heat capacity of the probes. We then use results from a finite-element model

6 to check the validity of the semianalytical solution and to assess whether it is appropriate to treat

7 the probes as perfect conductors. We conclude with a discussion of published data indicating that

8 thermal property and water content estimates may be biased if the DPHP method is implemented

9 without accounting for the finite properties of the probes. We also briefly discuss potential

10 limitations of the semianalytical solution.

\section{GENERAL SOLUTION}

13 Consider a DPHP sensor with infinitely long heater and temperature probes of finite radius,

14 finite heat capacity, and infinite thermal conductivity. The heater probe has radius $a_{1}$ and

15 volumetric heat capacity $C_{1}$, and the temperature probe has radius $a_{2}$ and volumetric heat

16 capacity $C_{2}$. The probes, with centerlines a distance $L$ apart and with $\left(a_{1}+a_{2}\right)<L$, are

17 surrounded by soil with volumetric heat capacity $C$ and thermal conductivity $\lambda$. We assume the

18 soil to be homogeneous and isotropic and that $C$ and $\lambda$ are not functions of temperature. We also

19 assume that the soil and probes are in perfect thermal contact. Let $V_{1}(t)$ and $V_{2}(t)$ be the

20 temperatures of the heater and temperature probes, respectively, where $t$ is time. We derive an

21 analytical solution in the Laplace domain for $V_{2}(t)$, given an arbitrary heating function $\phi(t)$ for

22 the heater probe. The function $\phi(t)$ gives the rate per unit length at which heat is released from

23 the heater probe. 
We begin by deriving an analytical solution in the Laplace domain that accounts only for the

2 finite radius and finite heat capacity of the heater probe; that is, we ignore the presence of the

3 temperature probe and consider a heater probe surrounded only by an infinite domain of soil.

4 This solution yields an expression for the transform of the temperature of the soil at a location

5 that coincides with the centerline of the temperature probe. That expression is then used to obtain

6 a solution for $V_{2}(t)$ in the Laplace domain that accounts for the finite radius and finite heat

7 capacity of the temperature probe.

\section{Heater Probe with Finite Properties}

10 The solution that accounts for only the heater probe is derived by using a coordinate system

11 (Fig. 1) with radial coordinate $r_{1}$ that is centered on the heater probe at $(x, y)=(0,0)$. The

12 temperature probe is located at $(x, y)=(L, 0)$. The distance from the centerline of the heater

13 probe to an arbitrary point in the $x-y$ domain is $r_{1}$, where $r_{1}^{2}=x^{2}+y^{2}$. The temperature of the

14 soil, $\mathrm{v}\left(r_{1}, t\right)$, satisfies the heat equation (Carslaw and Jaeger, 1959)

$$
\frac{\partial^{2} v}{\partial r_{1}^{2}}+\frac{1}{r_{1}} \frac{\partial v}{\partial r_{1}}-\frac{1}{\kappa} \frac{\partial v}{\partial t}=0 ; \quad a_{1} \leq r_{1}<\infty, t>0
$$

16 where $\kappa=\lambda / C$ is the thermal diffusivity. The boundary and initial conditions to be satisfied are

$$
v\left(r_{1}, t\right) \rightarrow 0 ; \quad r_{1} \rightarrow \infty, t>0
$$

$$
v\left(r_{1}, t\right)=0 ; \quad a_{1} \leq r_{1}<\infty, t=0
$$

$$
v\left(r_{1}, t\right)=V_{1}(t) ; \quad r_{1}=a_{1}, t>0
$$$$
-2 \pi a_{1} \lambda \frac{\partial v}{\partial r_{1}}=\phi(t)-\pi a_{1}^{2} C_{1} \frac{d V_{1}}{d t} ; \quad r_{1}=a_{1}, t>0
$$ 
1 Equation [5], the boundary condition for a medium in contact with a perfect conductor (Carslaw

2 and Jaeger, 1959, p. 22), is obtained by writing an energy balance for the heater probe. The left-

3 hand side of Eq. [5] is the total flux of heat per unit length from the heater probe into the soil at

$4 \quad r_{1}=a_{1}$. This problem is similar to one addressed in $\S 13.7$ II of Carslaw and Jaeger (1959), but

5 considers the more general case of an arbitrary heating function.

6 The Laplace domain solution for $\mathrm{v}\left(r_{1}, t\right)$ is obtained by making use of the definition

$$
\hat{v}\left(r_{1}, p\right) \equiv \int_{0}^{\infty} v\left(r_{1}, t\right) \exp (-p t) d t
$$

8 where $\hat{v}\left(r_{1}, p\right)$ is the Laplace transform of $v\left(r_{1}, t\right)$ and $p$ is the transform variable. Upon taking

9 the transforms of Eqs. [1] - [6], we obtain the subsidiary equation

$$
\frac{d^{2} \hat{v}}{d r_{1}^{2}}+\frac{1}{r_{1}} \frac{d \hat{v}}{d r_{1}}-\mu^{2} \hat{v}=0 ; \quad a_{1} \leq r_{1}<\infty
$$

11 and the boundary conditions

$$
\begin{gathered}
\hat{\mathrm{v}}\left(r_{1}, p\right) \rightarrow 0 ; \quad r_{1} \rightarrow \infty \\
\hat{\mathrm{v}}\left(r_{1}, p\right)=\hat{V}_{1}(p) ; \quad r_{1}=a_{1} \\
-2 \pi a_{1} \lambda \frac{d \hat{\mathrm{v}}}{d r_{1}}=\hat{\phi}(p)-p \pi a_{1}^{2} C_{1} \hat{V}_{1}(p) ; \quad r_{1}=a_{1}
\end{gathered}
$$

15 where

$$
\mu=\sqrt{p / \kappa}
$$

17 The radially symmetric solution of Eq. [8] that satisfies Eqs. [9] and [10] is

$$
\hat{v}\left(r_{1}, p\right)=\hat{V}_{1}(p) \frac{K_{0}\left(\mu r_{1}\right)}{K_{0}\left(\mu a_{1}\right)}
$$


1 where $K_{n}(\cdot)$ denotes the modified Bessel function of the second kind of order $n$. The remaining

2 boundary condition is satisfied by differentiating this expression with respect to $r_{1}$ and

3 substituting the result into Eq. [11] to give

$$
p \pi a_{1}^{2} C_{1} \hat{V}_{1}(p)=\hat{\phi}(p)-2 \pi \lambda \mu a_{1} \hat{V}_{1}(p) \frac{K_{1}\left(\mu a_{1}\right)}{K_{0}\left(\mu a_{1}\right)}
$$

$5 \quad$ Rearrangement of this expression yields

$$
\hat{V}_{1}(p)=\frac{\hat{\phi}(p) K_{0}\left(\mu a_{1}\right)}{2 \pi \lambda \mu a_{1}\left[K_{1}\left(\mu a_{1}\right)+\left(\mu a_{1} \beta_{1} / 2\right) K_{0}\left(\mu a_{1}\right)\right]}
$$

7 where $\beta_{1}=C_{1} / C$. Now that we have an expression for the temperature of the heater probe in the

8 transform domain, it can be substituted into Eq. [13] to give

$$
\hat{v}\left(r_{1}, p\right)=\frac{\hat{\phi}(p) K_{0}\left(\mu r_{1}\right)}{2 \pi \lambda \mu a_{1}\left[K_{1}\left(\mu a_{1}\right)+\left(\mu a_{1} \beta_{1} / 2\right) K_{0}\left(\mu a_{1}\right)\right]} ; \quad r_{1} \geq a_{1}
$$

10 which is the transform of the temperature field in the soil at a distance $r_{1}$ from the center of the

11 heater probe. This solution is the general case (i.e., the case for an arbitrary heating rate) of the

12 solution for continuous heating given in §13.7 II of Carslaw and Jaeger (1959).

13 Note that Eq. [16] is the product of the transfer function

$$
\hat{\mathrm{v}}_{f}\left(p, a_{1}, \beta_{1}\right)=\frac{1}{\mu a_{1}\left[K_{1}\left(\mu a_{1}\right)+\left(\mu a_{1} \beta_{1} / 2\right) K_{0}\left(\mu a_{1}\right)\right]}
$$

15 and Eq. [A3], which is the transform of a general line-source solution. Equation [16] can

16 therefore be written in the form

$$
\hat{\mathrm{v}}\left(r_{1}, p\right)=\hat{\mathrm{v}}_{f}\left(p, a_{1}, \beta_{1}\right) \frac{\hat{\phi}(p) K_{0}\left(\mu r_{1}\right)}{2 \pi \lambda} ; \quad r_{1} \geq a_{1}
$$

18 It is of interest that the transfer function $\hat{v}_{f}\left(p, a_{1}, \beta_{1}\right)$ does not depend on the functional form of

$19 \phi(t)$. The Laplace transform of the solution $v\left(r_{1}, t\right)$ at a distance $r_{1}=L$ is 


$$
\hat{v}(L, p)=\hat{v}_{f}\left(p, a_{1}, \beta_{1}\right) \frac{\hat{\phi}(p) K_{0}(\mu L)}{2 \pi \lambda}
$$

\section{Heater and Temperature Probes with Finite Properties}

We now use Eq. [19] to derive a Laplace-domain solution for $V_{2}(t)$, the temperature of the temperature probe. The solution is obtained by using an addition theorem for Bessel function

6 solutions of the heat equation. In implementing this approach, we make the simplifying assumption that $V_{2}(t)$ is influenced by the heater probe, but that $V_{1}(t)$ is not influenced by the

8 temperature probe. In other words, we assume that the temperature probe does not alter the radial

9 symmetry of the temperature distribution around the heater probe. This assumption is appropriate

10 if the radius of the probes is small relative to their distance of separation. We examine its validity

11 later by using results from a numerical model that does not impose a radially-symmetric

12 temperature distribution in the vicinity of the heater probe.

13 The solution is obtained by using a second coordinate system (Fig. 1) with radial coordinate

$14 r_{2}$ that is centered on the temperature probe at $(x, y)=(L, 0)$. The distance from the centerline of

15 the temperature probe to an arbitrary point in the $x-y$ domain is $r_{2}$, where $r_{2}^{2}=(x-L)^{2}+y^{2}$. In

16 terms of the new coordinates, the heat equation takes the form

$$
\frac{\partial^{2} v}{\partial r_{2}^{2}}+\frac{1}{r_{2}} \frac{\partial v}{\partial r_{2}}+\frac{1}{r_{2}^{2}} \frac{\partial^{2} v}{\partial \theta^{2}}-\frac{1}{\kappa} \frac{\partial v}{\partial t}=0 ; \quad a_{2} \leq r_{2}<\infty, 0 \leq \theta<2 \pi, t>0
$$

18 where the angle $\theta$ is such that $(x-L, y)=\left(r_{2} \cos \theta, r_{2} \sin \theta\right)$. The boundary and initial conditions

19 to be satisfied are

$$
\mathrm{v}\left(r_{2}, \theta, t\right)=V_{2}(t) ; \quad r_{2}=a_{2}, 0 \leq \theta<2 \pi, t>0
$$

$$
a_{2} \lambda \int_{0}^{2 \pi} \frac{\partial v}{\partial r_{2}} d \theta=\pi a_{2}^{2} C_{2} \frac{d V_{2}}{d t} ; \quad r_{2}=a_{2}, t>0
$$




$$
V_{2}(t)=0 ; \quad t=0
$$

2 Taking the Laplace transform of these expressions yields the subsidiary equation

$$
\frac{d^{2} \hat{v}}{d r_{2}^{2}}+\frac{1}{r_{2}} \frac{d \hat{v}}{d r_{2}}+\frac{1}{r_{2}^{2}} \frac{d^{2} \hat{v}}{d \theta^{2}}-\mu^{2} \hat{v}=0 ; \quad a_{2} \leq r_{2}<\infty, 0 \leq \theta<2 \pi
$$

4 and the boundary conditions

$$
\lambda a_{2} \int_{0}^{2 \pi} \frac{d \hat{v}}{d r_{2}} d \theta=p \pi a_{2}^{2} C_{2} \hat{V}_{2}(p) ; \quad r_{2}=a_{2}
$$

To obtain the desired solution, we consider the spatial dependence $K_{0}\left(\mu r_{1}\right)$ of the transform

8 of the heat emitted by the heater probe and use an addition theorem for Bessel function solutions

9 of Eq. [24] (Carslaw and Jaeger, 1959, p. 377, Eq. [1]) to write $K_{0}\left(\mu r_{1}\right)$ in terms of solutions

$10 I_{m}\left(\mu r_{2}\right) \cos (m \theta)$ of Eq. [24] centered at the temperature probe, where $I_{m}(\cdot)$ denotes the

11 modified Bessel function of the first kind of order $m$. For $m \neq 0$ these solutions are not radially

12 symmetric. The addition theorem for $r_{2}<L$ is

$$
K_{0}\left(\mu r_{1}\right)=\sum_{m=-\infty}^{m=\infty}(-1)^{m} K_{m}(\mu L) I_{m}\left(\mu r_{2}\right) \cos (m \theta)
$$

14 We look for a solution $\hat{v}\left(r_{2}, \theta, p\right)$ of Eq. [24] valid for $a_{2}<r_{2}<L$ that satisfies

$15 \hat{v}\left(r_{2}, \theta, p\right)=\hat{V}_{2}(p)$. That is, we look for a solution of the form

$$
\hat{\mathrm{v}}\left(r_{2}, \theta, p\right)=\hat{V}_{2}(p) \frac{K_{0}\left(\mu r_{2}\right)}{K_{0}\left(\mu a_{2}\right)}+\hat{\phi}(p) \hat{\mathrm{v}}_{f}\left(p, a_{1}, \beta_{1}\right) \frac{K_{0}\left(\mu r_{1}\right)}{2 \pi \lambda}-\sum_{m=-\infty}^{m=\infty}(-1)^{m} d_{m} K_{m}\left(\mu r_{2}\right) \cos (m \theta)
$$

17 with coefficients $d_{m}$ to be determined by satisfying Eq. [25]. This requires that

$$
d_{m}=\hat{\phi}(p) \hat{v}_{f}\left(p, a_{1}, \beta_{1}\right) \frac{K_{m}(\mu L) I_{m}\left(\mu a_{2}\right)}{2 \pi \lambda K_{m}\left(\mu a_{2}\right)}
$$


1 Upon substituting Eqs. [27] and [29] into Eq. [28], the solution $\hat{v}\left(r_{2}, \theta, p\right)$ takes the form

$$
\begin{aligned}
\hat{\mathrm{v}}\left(r_{2}, \theta, p\right) & =\hat{V}_{2}(p) \frac{K_{0}\left(\mu r_{2}\right)}{K_{0}\left(\mu a_{2}\right)}+\hat{\phi}(p) \frac{\hat{\mathrm{v}}_{f}\left(p, a_{1}, \beta_{1}\right)}{2 \pi \lambda} \sum_{m=-\infty}^{m=\infty}(-1)^{m} K_{m}(\mu L) I_{m}\left(\mu r_{2}\right) \cos (m \theta) \\
& -\hat{\phi}(p) \frac{\hat{\mathrm{v}}_{f}\left(p, a_{1}, \beta_{1}\right)}{2 \pi \lambda} \sum_{m=-\infty}^{m=\infty}(-1)^{m} \frac{K_{m}(\mu L) I_{m}\left(\mu a_{2}\right) K_{m}\left(\mu r_{2}\right) \cos (m \theta)}{K_{m}\left(\mu a_{2}\right)}
\end{aligned}
$$

3 To satisfy the remaining boundary condition, we differentiate Eq. [30] with respect to $r_{2}$ and

4 substitute the result into Eq. [26]. After evaluating the integral, Eq. [26] becomes

$$
\begin{aligned}
p \pi a_{2}^{2} C_{2} \hat{V}_{2}(p)= & -2 \pi \lambda \mu a_{2} \hat{V}_{2}(p) \frac{K_{1}\left(\mu a_{2}\right)}{K_{0}\left(\mu a_{2}\right)}+\mu a_{2} \hat{\phi}(p) \hat{v}_{f}\left(p, a_{1}, \beta_{1}\right) K_{0}(\mu L) I_{1}\left(\mu a_{2}\right) \\
& +\mu a_{2} \hat{\phi}(p) \hat{v}_{f}\left(p, a_{1}, \beta_{1}\right) \frac{K_{0}(\mu L) I_{0}\left(\mu a_{2}\right) K_{1}\left(\mu a_{2}\right)}{K_{0}\left(\mu a_{2}\right)}
\end{aligned}
$$

6 Comparing this expression with Eq. [30] reveals that the terms periodic in $\theta$ make no net

7 contribution to the total heat flux per unit length at $r_{2}=a_{2}$. Equation [31] contains only the

8 contribution from the terms for $m=0$ in Eq. [27]. We now use the Wronskian relation (Olver,

$9 \quad 1965$, p. 375)

$$
I_{0}\left(\mu a_{2}\right) K_{1}\left(\mu a_{2}\right)+I_{1}\left(\mu a_{2}\right) K_{0}\left(\mu a_{2}\right)=1 /\left(\mu a_{2}\right)
$$

11 to write Eq. [31] in the form

$$
p \pi a_{2}^{2} C_{2} \hat{V}_{2}(p) K_{0}\left(\mu a_{2}\right)=-2 \pi \lambda \mu a_{2} \hat{V}_{2}(p) K_{1}\left(\mu a_{2}\right)+\hat{\phi}(p) \hat{v}_{f}\left(p, a_{1}, \beta_{1}\right) K_{0}(\mu L)
$$

13 Rearrangement of this result yields the expression

$$
\hat{V}_{2}(p)=\frac{\hat{\phi}(p) \hat{v}_{f}\left(p, a_{1}, \beta_{1}\right) K_{0}(\mu L)}{2 \pi \lambda \mu a_{2}\left[K_{1}\left(\mu a_{2}\right)+\left(\mu a_{2} \beta_{2} / 2\right) K_{0}\left(\mu a_{2}\right)\right]}
$$

15 where $\beta_{2}=C_{2} / C$.

16 The solution in the transform domain for the temperature of the temperature probe is then

$$
\hat{V}_{2}(p)=\hat{v}_{f}\left(p, a_{1}, \beta_{1}\right) \hat{v}_{f}\left(p, a_{2}, \beta_{2}\right) \frac{\hat{\phi}(p) K_{0}(\mu L)}{2 \pi \lambda}
$$


1 where the transfer functions corresponding to heater and temperature probes have exactly the

2 same form. Note that Eq. [35] is Eq. [A3], the transform of a general line-source solution,

3 multiplied by the product of the transfer functions for the heater and temperature probes. With

$4 \hat{v}_{f}\left(p, a_{2}, \beta_{2}\right)=1$, Eq. [35] is identical to Eq. [19] and gives the transform of the solution for the

5 case where the heater probe has finite radius and finite heat capacity but the temperature probe

6 has zero radius. With $\hat{v}_{f}\left(p, a_{1}, \beta_{1}\right)=1$, Eq. [35] gives the transform of the solution for the case

7 where the temperature probe has finite radius and finite heat capacity but the heater probe has

8 zero radius.

9 In deriving Eq. [35], we assumed that the soil and both probes have a temperature of zero at

10 time $t=0$. Employing this initial condition does not result in a loss of generality. The principle

11 of superposition permits application of this solution to cases where the soil and both probes have

12 arbitrary uniform initial temperature $\mathrm{v}_{0}$, for which we have $\mathrm{v}\left(r_{1}, 0\right)=V_{1}(0)=V_{2}(0)=\mathrm{v}_{0}$. For such

13 cases $V_{2}(t)$ represents the temperature rise above the initial temperature $v_{0}$.

16 For most applications, the probes of the DPHP sensor have the same radius and the same heat

17 capacity. If both have radius $a_{0}$ and heat capacity $C_{0}$, we have $\beta_{0}=C_{0} / C$, and the transfer

18 function for the probes can be written as

$$
\hat{v}_{f}\left(p, a_{0}, \beta_{0}\right)=\frac{1}{\mu a_{0}\left[K_{1}\left(\mu a_{0}\right)+\left(\mu a_{0} \beta_{0} / 2\right) K_{0}\left(\mu a_{0}\right)\right]}
$$

This simplification allows us to write Eq. [35] in the form

$$
\hat{V}_{2}(p)=\hat{v}_{f}^{2}\left(p, a_{0}, \beta_{0}\right) \frac{\hat{\phi}(p) K_{0}(\mu L)}{2 \pi \lambda}
$$


The appropriate heating function for DPHP applications is

$$
\phi(t)=\left\{\begin{array}{l}
q^{\prime} ; \quad 0<t \leq t_{0} \\
0 ; \quad t>t_{0}
\end{array}\right.
$$

3 where $q^{\prime}$ is the rate per unit length at which heat is released from the heater probe and $t_{0}$ is the

4 heating duration. The Laplace transform of Eq. [38] is $\hat{\phi}(p)=q^{\prime}\left[1-\exp \left(-p t_{0}\right)\right] / p$. Substituting

5 this result into Eq. [35] yields

$$
\hat{V}_{2}^{\mathrm{P}}(p)=\hat{v}_{f}^{2}\left(p, a_{0}, \beta_{0}\right) \frac{q^{\prime}\left[1-\exp \left(-p t_{0}\right)\right] K_{0}(\mu L)}{2 \pi \lambda p}
$$

7 where the superscript "P" indicates that this expression is the Laplace-domain solution for the 8 case of pulsed heating. Equation [39] is the product of $\hat{v}_{f}^{2}\left(p, a_{0}, \beta_{0}\right)$ and the transform of Eq.

9 [A1], the line-source solution used in the DPHP method of Bristow et al. (1994). This solution

10 can be inverted numerically to obtain values of $V_{2}^{\mathrm{P}}(t)$ for times of interest, but better accuracy

11 can be achieved by performing the numerical inversion for the case of continuous heating, and

12 then using the principle of superposition in the time domain to account for the finite duration of

13 heating. This requires that we use $\phi(t)=q^{\prime}$, which has the form $\hat{\phi}(p)=q^{\prime} / p$ in the transform

14 domain. Thus, Eq. [35] becomes

$$
\hat{V}_{2}^{\mathrm{C}}(p)=\hat{v}_{f}^{2}\left(p, a_{0}, \beta_{0}\right) \frac{q^{\prime} K_{0}(\mu L)}{2 \pi \lambda p}
$$

16 for the case of continuous heating. Equation [40a] can be inverted numerically to obtain values

17 of $V_{2}^{\mathrm{C}}(t)$ and $V_{2}^{\mathrm{C}}\left(t-t_{0}\right)$ for particular times of interest. These values of $V_{2}^{\mathrm{C}}(t)$ and $V_{2}^{\mathrm{C}}\left(t-t_{0}\right)$ are

18 then used to obtain the corresponding pulsed heating results, $V_{2}^{\mathrm{P}}(t)$, by using the expression

$$
V_{2}^{\mathrm{P}}(t)=\left\{\begin{array}{l}
V_{2}^{\mathrm{C}}(t) ; \quad 0<t \leq t_{0} \\
V_{2}^{\mathrm{C}}(t)-V_{2}^{\mathrm{C}}\left(t-t_{0}\right) ; \quad t>t_{0}
\end{array}\right.
$$


1 Together, Eqs. [40a] and [40b] represent a semianalytical solution for the temperature of the

2 temperature probe. Hereafter, we refer to Eq. [40] as the identical-cylindrical-perfect-conductors

3 (ICPC) solution.

4 An important property of the ICPC solution is that it becomes identical to the line-source

5 solution of Bristow et al. (1994) when $\hat{v}_{f}\left(p, a_{0}, \beta_{0}\right)=1$, which corresponds to the case where the

6 heater probe and temperature probe both have zero radius. Thus, the effects of the finite radius

7 and finite heat capacity of the probes can be examined by comparing values of $V_{2}^{\mathrm{P}}(t)$ from Eq.

8 [40] with values of $\mathrm{v}(L, t)$ obtained by evaluating Eq. [A1] with $r_{1}=L$. That approach was used

9 to obtain the results presented in the next section. The ICPC solution and Eq. [A1] were

10 evaluated using MATLAB (ver. 7.8, The MathWorks, Inc., Natick, MA). The modified Bessel

11 functions of the second kind and the exponential integral were evaluated using the built-in

12 functions BESSELK and EXPINT, respectively. Numerical inversion of Eq. [40a] was

13 performed by using the algorithm of Stehfest $(1970 a, b)$ with 16 coefficients. Details of the

14 numerical inversion procedure are given in Appendix B. A copy of the MATLAB script used to

15 perform these calculations is available upon request.

\section{INFLUENCE OF THE PROBES}

18 A key result evident from the functional form of the ICPC solution is that the finite properties

19 of the heater probe and the finite properties of the temperature probe have equal influence in

20 modifying the heat-pulse signal received by the temperature probe. This follows from the fact

21 that both probes have the same transfer function. If one of the probes has zero radius, Eq. [40a]

22 takes the form 


$$
\hat{V}_{2}^{\mathrm{C}}(p)=\hat{v}_{f}\left(p, a_{0}, \beta_{0}\right) \frac{q^{\prime} K_{0}(\mu L)}{2 \pi \lambda p}
$$

2 regardless of whether that probe is the heater probe or the temperature probe. Therefore, having a

3 heater probe of finite radius and a temperature probe of zero radius produces the same result in

4 the time domain as having a heater probe of zero radius and a temperature probe of finite radius.

5 This result is consistent with the spatial sensitivity of the DPHP method worked out by Knight et

6 al. (2007). They showed that the spatial sensitivity in the vicinity of the temperature probe is

7 identical to that in the vicinity of the heater probe. If we consider the probes to be heterogeneities

8 with a heat capacity slightly different from that of the soil, the spatial sensitivity of the DPHP

9 method indicates that both "heterogeneities" must have equal influence on the heat-pulse signal

10 received by the temperature probe. Hereafter, we consider only the combined effects of the

11 heater and temperature probes. Despite the fact that both probes have equal influence, their

12 combined effect in the time domain is not simply additive because it is the result of convolution

13 operations.

14 In the remainder of this section we use the ICPC and line-source solutions to examine the

15 effect of the finite radius of the probes and the effect of the finite heat capacity of the probes. The

16 dimensionless quantities that primarily determine the extent of these two effects are $a_{0} / L$ and

$17 \beta_{0}$. We first present results for arbitrary values of $a_{0} / L$ and $\beta_{0}$ to isolate the effects of finite

18 radius and finite heat capacity. We then present results obtained by using values of $a_{0} / L$ and $\beta_{0}$

19 for a typical DPHP sensor.

\section{Finite Radius}

22 The effect of the finite radius of the probes was examined by evaluating the ICPC solution 
1 with $\beta_{0}=1$ and ratios $a_{0} / L$ of $0.05,0.1$, and 0.15 . By definition, the heat capacity of the probes

2 is identical to that of the soil when $\beta_{0}=1$. The calculations were performed using $q^{\prime}=100 \mathrm{~W}$

$3 \mathrm{~m}^{-1}, t_{0}=8 \mathrm{~s}, L=6.0 \times 10^{-3} \mathrm{~m}, C=2.0 \mathrm{MJ} \mathrm{m}^{-3} \mathrm{~K}^{-1}, \lambda=0.5 \mathrm{~W} \mathrm{~m}^{-1} \mathrm{~K}^{-1}$, and values for $a_{0}$ and $C_{0}$

4 that yielded the desired values for $a_{0} / L$ and $\beta_{0}$. The same values for $q^{\prime}, t_{0}, L, C$, and $\lambda$ were used

5 to evaluate the line-source solution. The results (Fig. 2) are plotted for the case of zero initial

6 temperature to be consistent our mathematical notation; however, as noted earlier, the results

7 also hold for the case of an arbitrary uniform temperature $v_{0}$. In that case, the curves in Fig. 2

8 represent the change in temperature from the initial temperature $v_{0}$.

9 Comparing the curves for the ICPC and line-source solutions (Fig. 2) shows that the finite

10 radius of the probes causes the heat-pulse signal to arrive at the temperature probe slightly earlier

11 in time. The magnitude of this time shift increases as the radius of the probes increases. In the

12 limit as $a_{0} \rightarrow 0$, the effect of finite probe radius vanishes and the ICPC solution becomes

13 identical to the line-source solution. This is consistent with the fact that $\hat{v}_{f}\left(p, a_{0}, \beta_{0}\right) \rightarrow 1$ as $a_{0}$

$14 \rightarrow 0$. For other combinations of the parameters $q^{\prime}, t_{0}, L, C$, and $\lambda$ there is essentially no change in

15 the way the effect of finite probe radius is manifested in the results; however, for a given probe

16 radius, the magnitude of the time shift is influenced by the thermal diffusivity and the probe

17 spacing. Specifically, the time shift decreases linearly with $\kappa$ and increases linearly with $L^{2}$.

18 The finite radius of the probes causes a time shift because the effective distance traveled by

19 the heat-pulse signal is smaller for the ICPC solution than for the line-source solution. The line-

20 source solution assumes that the heat pulse originates at the origin and that the temperature rise is

21 recorded at $r_{1}=L$. Thus, the effective travel distance is $L$. In contrast, the effective travel

22 distance for the ICPC solution lies somewhere between $L$ and $L-2 a_{0}$ because the heat pulse 
1 originates at $r_{1}=a_{0}$ and the signal is received at $r_{2}=a_{0}$. The effective travel distance clearly

2 decreases with increasing probe radius.

3 Although the ICPC solution accounts for the finite radius of the heater and temperature

4 probes, it does so only approximately because the probes are considered to be perfect conductors.

5 By not accounting for the finite conductivity of the probes, the ICPC solution overestimates the

6 time-shift caused by the finite radius of the probes. It follows that this solution will be useful for

7 DPHP applications only if the overestimation of this time-shift is minimal. In other words, the

8 ICPC solution will be useful only if it allows the effect of finite probe radius to be approximated

9 with sufficient accuracy. This will be the case if the thermal conductivity of the probes is

10 sufficiently large relative to the conductivity of the soil. We examine this issue in detail later by

11 using results from a numerical model that accounts for the finite conductivity as well as the finite

12 heat capacity of the probes.

\section{Finite Heat Capacity}

15 The effect of the finite heat capacity of the probes was examined by evaluating the ICPC

16 solution with $a_{0} / L=0.1$ and $\beta_{0}$ values of $0.5,1$, and 2 . Calculations were performed using the

17 same parameter values that were used in the previous section. The influence of the finite heat

18 capacity of the probes can be understood by comparing the curves for $\beta_{0}=0.5$ and $\beta_{0}=2$ with

19 the curve for $\beta_{0}=1$ (Fig. 3). Less energy is needed to raise the temperature of the probes when

$20 \beta_{0}<1$. This results in an increase in the magnitude of the heat-pulse signal, and the signal is

21 positively skewed so the maximum temperature rise occurs earlier. Conversely, when $\beta_{0}>1$,

22 more energy is needed to raise the temperature of the probes than for the case where $\beta_{0}=1$. As a 
1 result, the magnitude of the heat-pulse signal decreases and the signal is negatively skewed so

2 the maximum temperature rise occurs later.

3 To understand why $\beta_{0}$ influences the skewness of the heat-pulse signal, it is useful to

4 consider the time rate of change of the heat flux at the interface between the heater probe and the

5 soil. The heat flux at $r_{1}=a_{0}$ increases rapidly with time following the onset of heating. Because

6 less energy is needed to raise the temperature of the heater probe when $\beta_{0}<1$, the heat flux at $r_{1}$

$7=a_{0}$ increases more sharply with time than for the case where $\beta_{0}=1$. This produces a signal that

8 is positively skewed relative to the signal for the case where $\beta_{0}=1$. The negatively skewed

9 signal for the case of $\beta_{0}>1$ is caused by a similar but opposite effect.

10 As noted earlier, the ICPC solution only approximately accounts for the effect of finite probe

11 radius because it does not account for the finite conductivity of the probes; however, it accounts

12 for the finite heat capacity of the probes in a way that is physically correct. Thus, the potential

13 utility of the ICPC solution for DPHP applications depends on whether the benefit gained by

14 accounting for the finite heat capacity of the probes offsets the loss of accuracy caused by

15 approximating the effect of finite probe radius.

\section{Results for Typical DPHP Sensor}

18 Here we present results obtained by evaluating the ICPC and line-source solutions using

19 values of $a_{0}$ and $C_{0}$ for the DPHP sensor of Basinger et al. (2003). Figure 4 shows a cross-section

20 of the probes of their sensor. The probes were fabricated from type 304 stainless-steel tubing

21 (0.828-mm i.d., 1.27-mm o.d.) that was filled with thermally conductive epoxy (Omegabond

22 101, Omega Engineering, Inc., Stamford, CT). The volumetric heat capacity of the probes can be

23 approximated as the weighted average 


$$
C_{0}=\left(a_{\mathrm{e}} / a_{0}\right)^{2} C_{\mathrm{e}}+\left[1-\left(a_{\mathrm{e}} / a_{0}\right)^{2}\right] C_{\mathrm{ss}}
$$

2 where $a_{\mathrm{e}}$ is the radius of the epoxy-filled region of the heater and temperature probes, $C_{\mathrm{e}}$ is the

3 volumetric heat capacity of the thermally conductive epoxy, and $C_{\mathrm{ss}}$ is the volumetric heat

4 capacity of the stainless steel. This expression yields $C_{0}=2.84 \mathrm{MJ} \mathrm{m}^{-3} \mathrm{~K}^{-1}$ for the parameter

5 values in Tables 1 and 2.

6 Results for the sensor of Basinger et al. (2003) were obtained for air-dried sand, water-

7 saturated sand, and water. The dry and wet sands were chosen because their conductivities bound

8 the range of conductivities typically encountered in mineral soils. Water was chosen because

9 sensor calibration (i.e., determination of apparent probe spacing) typically involves a

10 measurement in water immobilized with agar (Campbell et al., 1991). The volumetric heat

11 capacities of these media (Table 1 ) give $\beta_{0}$ values of $2.58,0.93$, and 0.68 for the dry sand, wet

12 sand, and water, respectively. Values for the parameters that remained fixed for these

13 calculations (Table 2 ) yield a radius/spacing ratio of $a_{0} / L \approx 0.11$.

14 Consider first the results for wet sand (Fig. 5), where the heat capacity of the probes is only 15 slightly smaller than that of the soil $\left(\beta_{0}=0.93\right)$. Because $\beta_{0} \approx 1$, the finite heat capacity of the

16 probes has minimal effect. The difference between the curves for the ICPC and line-source

17 solutions is primarily due to the finite radius of the probes. Accounting for the finite radius of the 18 probes produces a heat-pulse signal that arrives approximately $0.35 \mathrm{~s}$ earlier. Although the effect

19 of finite probe radius for the dry sand $\left(\beta_{0}=2.58\right)$ and water $\left(\beta_{0}=0.68\right)$ cannot be determined by

20 inspecting results in Fig. 5, it can be estimated by considering the diffusivities of the three media.

21 The time shift for the dry sand is approximately $0.8 \mathrm{~s}$ because the diffusivity of the dry sand is

22 smaller than that of the wet sand by a factor of about 2.3. Similarly, the time shift for water is

23 approximately $1.5 \mathrm{~s}$ because the diffusivity of water is about 4.4 times smaller than that of the 
1 wet sand. For the dry sand, the leftward shift caused by the finite radius of the probes is offset by

2 a substantial effect due to their finite heat capacity (Fig. 5). Combined, the two effects reduce the

3 maximum temperature rise by about $0.15 \mathrm{~K}$ and delay the arrival time of the maximum

4 temperature rise by approximately $5 \mathrm{~s}$. In contrast, for water, the two effects combine to increase

5 the maximum temperature rise by about $0.09 \mathrm{~K}$, and they cause the maximum temperature rise to

6 appear approximately $4 \mathrm{~s}$ earlier.

7 The results presented in Fig. 5 clearly indicate that the DPHP method will yield biased

8 estimates of thermal properties if the line-source solution is used for thermal property estimation.

9 To quantify this bias, we determined the thermal properties required to force the line-source

10 solution into agreement with the curves in Fig. 5 that were generated with the ICPC solution.

11 This was accomplished by determining the maximum temperature rise, $V_{2}\left(t_{\mathrm{m}}\right)$, and the time at

12 which it occurred, $t_{\mathrm{m}}$, for each of the three curves generated with the ICPC solution. These pairs

13 of $V_{2}\left(t_{\mathrm{m}}\right)$ and $t_{\mathrm{m}}$ values were then substituted into Eq. [8] - [10] of Bristow et al. (1994) to

14 determine thermal properties using line-source theory. The results for dry sand (Table 3) show

15 that, if the finite radius and finite heat capacity of the probes are not taken into account, heat

16 capacity is overestimated by $6.4 \%$, and the conductivity and diffusivity are underestimated by

$17 \quad 7.7 \%$ and $13.5 \%$, respectively. Ignoring the finite properties of the probes has less of an effect on

18 the thermal properties of wet sand and water (Table 3), but the errors in the conductivity and

19 diffusivity of water are sufficiently large to be of concern. Clearly, if the ICPC solution provides

20 a reasonable approximation of reality, the results presented here for a typical DPHP sensor show

21 that the effects of the finite radius and finite heat capacity of the probes may be substantial,

22 especially for the case where there is a large contrast between the heat capacity of the probes and

23 that of the soil. 
2 In this section we use results from a numerical model to check the validity of the ICPC 3 solution and to assess whether it is appropriate to treat the probes as perfect conductors. We

4 begin with a description of the numerical model.

\section{Numerical Model}

7 In the numerical model, we relax the constraint on thermal conductivity and consider probes

8 of finite radius that have finite conductivity as well as finite heat capacity. We also account for

9 the fact that the heater and temperature probes are composite solids that consist of stainless steel

10 and thermally conductive epoxy (Fig. 4). As in the derivation of the semianalytical solution, we

11 consider both probes to be infinite in length and we model heat transfer in a plane normal to the

12 axis of the probes that coincides with the location of the thermistor. The heater probe is centered

13 at the origin and the temperature probe is centered at $(x, y)=(L, 0)$, as in Fig. 1. Both probes

14 have radius $a_{0}$ and epoxy-filled regions of radius $a_{\mathrm{e}}$. We do not account for the finite thermal

15 properties of the thermistor and electrical resistance wire, effectively assuming that they have

16 thermal properties identical to those of the epoxy. The problem domain is the semicircular region

17 defined by $0 \leq r_{1} \leq a_{\mathrm{b}}$ and $0 \leq \theta \leq \pi$. We assume no contact resistance at the material interfaces

18 within this domain, and that the thermal properties $C$ and $\lambda$ within each portion of the domain are

19 homogeneous, isotropic, and independent of temperature.

20 The temperature $T(x, y, t)$ in the problem domain satisfies the heat equation

$$
C \frac{\partial T}{\partial t}=\nabla \cdot(\lambda \nabla T)+g ; \quad t>0
$$


1 where $g(x, y, t)$ is the rate of heat generation per unit volume and $r_{1}^{2}=x^{2}+y^{2}$. The heat

2 equation was solved with a zero initial condition and with temperature $T=0$ on the external

3 boundary at $r_{1}=a_{\mathrm{b}}$. The radius $a_{\mathrm{b}}$ was made sufficiently large that the solution is effectively the

4 solution for a boundary condition of $T \rightarrow 0$ as $r \rightarrow \infty$. Owing to symmetry considerations, a zero-

5 flux condition was imposed along the external boundary coincident with the $x$ axis. The solution

6 of Eq. [43] was also subject to temperature continuity and normal heat flux continuity conditions

7 at all internal boundaries (i.e., at material interfaces). Because the heater probe is usually

8 modeled as a line source of infinite length, it is customary to quantify the heat generation for a

9 DPHP measurement in terms of $q^{\prime}$, the rate of energy released per unit length of a line source.

10 Here we assume that the heat released by the four strands of resistance wire (Fig 4) is uniformly

11 distributed over the cross-section of the epoxy in the heater probe. Thus, the rate of heat

12 generation is represented by

$$
g(x, y, t)=\left\{\begin{array}{l}
q^{\prime} /\left(\pi a_{\mathrm{e}}^{2}\right) ; \quad 0 \leq r_{1}<a_{\mathrm{e}}, \quad 0 \leq \theta<\pi, \quad 0<t \leq t_{0} \\
0 ; \quad 0 \leq r_{1}<a_{\mathrm{e}}, \quad 0 \leq \theta<\pi, \quad t>t_{0} \\
0 ; \quad r_{1}>a_{\mathrm{e}}, \quad 0 \leq \theta<\pi, \quad t>0
\end{array}\right.
$$

14 The boundary value problem described above was solved with COMSOL Multiphysics

15 finite-element software (Version 3.5a, COMSOLAB, Stockholm, Sweden). All simulations were

16 performed with a domain radius of $a_{\mathrm{b}}=6.0 \times 10^{-2} \mathrm{~m}$ and a mesh that consisted of 12,246

17 triangular elements. Element size ranged from approximately $2.4 \times 10^{-6} \mathrm{~m}^{2}$ at the perimeter of

18 the domain to approximately $4.7 \times 10^{-10} \mathrm{~m}^{2}$ along the interfaces between the thermally

19 conductive epoxy and the stainless steel tubing (Fig. 6). The temperature $T(L, 0, t)$ at

$20(x, y)=(L, 0)$ was taken to represent the temperature rise recorded by thermistor. 
2 In deriving the ICPC solution, we assumed that the temperature distribution in the vicinity of

3 the heater probe is radially symmetric. For this assumption to hold, the ratio $a_{0} / L$ must be

4 sufficiently small that the temperature probe has negligible effect on the temperature distribution

5 near the heater probe. Obviously, this condition must be satisfied if the ICPC solution is to

6 provide a good approximation of the temperature probe temperature for the case where the both

7 probes have infinite conductivity. In a forthcoming paper we will examine the validity of the

8 solution in greater detail. Here we address the validity of the solution only for the DPHP sensor

9 of Basinger et al. (2003), for which $a_{0} / L \approx 0.11$.

10 Evaluating the validity of the ICPC solution requires a model that treats the probes as perfect

11 conductors and allows for a temperature distribution in the vicinity of the heater probe that is not

12 radially symmetric. This was achieved with our numerical model by assigning an arbitrarily large

13 value of conductivity for the portions of the domain containing epoxy or stainless steel. Results

14 were obtained for a conductivity of $1.0 \times 10^{5} \mathrm{~W} \mathrm{~m}^{-1} \mathrm{~K}^{-1}$, which is more than 6,700 times greater

15 than the conductivity of the stainless steel. All simulations were performed using values for $C_{\mathrm{e}}$

16 and $C_{\mathrm{ss}}$ from Table 1 and the values for $a_{\mathrm{e}}, a_{0}, q^{\prime}, t_{0}$ and $L$ given in Table 2 . Results for dry sand,

17 wet sand, and water were obtained by using the soil thermal properties given Table 1.

18 For all three media, the numerically simulated temperature rise curves, $T(L, 0, t)$, are nearly

19 identical to the curves for the ICPC solution shown in Fig. 5. We compare results from the two

20 solutions by examining the difference $V_{2}^{\mathrm{P}}(t)-T(L, 0, t)$ as a function of time (Fig. 7). Agreement

21 between the analytical and numerical results was best for the wet sand and water (Fig. 7), with

22 values for $V_{2}^{\mathrm{P}}(t)-T(L, 0, t)$ no greater than $1.4 \times 10^{-4} \mathrm{~K}$ and $8.6 \times 10^{-5} \mathrm{~K}$ for the wet sand and 
1 water, respectively. Thus, for the wet sand and water, it appears that $a_{0} / L \approx 0.11$ is sufficiently

2 small that the ICPC solution provides an excellent approximation of the temperature of the

3 temperature probe for the case where both probes are perfect conductors. Agreement between the

4 analytical and numerical solutions was not as good for the dry sand (Fig. 7). The assumption of a

5 radially symmetric temperature distribution near the heater probe was not satisfied to the same

6 extent for dry sand as for wet sand and water. Nevertheless, the magnitude of the difference

7 between the analytical and numerical results (no greater than $7.6 \times 10^{-4} \mathrm{~K}$ ) suggests that the

8 ICPC solution approximates the temperature of the temperature probe with sufficient accuracy to

9 be of use for DPHP applications where $a_{0} / L \leq 0.11$.

\section{Appropriateness of Solution for DPHP Applications}

12 Although the ICPC solution closely approximates the temperature of the temperature probe

13 for the case where both probes are perfect conductors, it will be appropriate for DPHP

14 applications only if it is appropriate to treat the probes as perfect conductors. Whereas the ICPC

15 solution correctly accounts for the finite heat capacity of the probes, it only approximately

16 accounts for their finite radius because it does not account for their finite conductivity. Thus, its

17 appropriateness for DPHP applications depends on whether the benefit gained by accounting for

18 finite heat capacity offsets the loss of accuracy caused by approximating the effect of finite

19 radius. To examine this issue, we performed simulations for the same three cases described in the

20 previous section, but with a version of the numerical model in which the probe materials had

21 finite conductivity as well as finite heat capacity. Simulations were performed using the

22 conductivities for epoxy and stainless steel given in Table 2 . We compared these results to

23 results obtained with both the ICPC solution and the line-source solution. The appropriateness of 
1 the ICPC solution is addressed by evaluating its performance relative to that of the line-source

2 solution, which does not account for the finite properties of the probes.

3 For all three media, the numerically simulated curves, $T(L, 0, t)$, are nearly identical to the

4 curves for the ICPC solution shown in Fig. 5. The level of agreement can be seen by examining

5 the difference $V_{2}^{\mathrm{P}}(t)-T(L, 0, t)$ as a function of time (Fig. 8). The difference curves for all three

6 media show that the ICPC solution overestimates the temperature of the temperature probe at

7 relatively early times and underestimates it at relatively late times. The reason for this trend is

8 addressed later in this section. Here we draw attention to the fact that the numerically simulated

9 values agree quite well with the values of $V_{2}^{\mathrm{P}}(t)$ from the ICPC solution. The magnitude of the

10 difference $V_{2}^{\mathrm{P}}(t)-T(L, 0, t)$ is no greater than $0.016 \mathrm{~K}$ for the dry sand, no greater than $0.012 \mathrm{~K}$

11 for the wet sand, and no greater than $0.004 \mathrm{~K}$ for water.

12 In contrast, the level of agreement between the numerically simulated curves and the line-

13 source solution is not nearly as good. This can be seen by examining the difference

$14 v(d, t)-T(L, 0, t)$ as a function of time (Fig. 9). The line-source solution overestimates the

15 temperature of the temperature probe by as much as $0.35 \mathrm{~K}$ for the dry sand, and underestimates

16 it by as much as $0.025 \mathrm{~K}$ and $0.056 \mathrm{~K}$ for the wet sand and water, respectively. Clearly, the ICPC

17 solution offers significant improvement over the line-source solution in characterizing the

18 temperature of the temperature probe. We therefore conclude that it is appropriate to treat the

19 probes of a DPHP sensor as perfect conductors for applications where $a_{0} / L \leq 0.11$.

20 Further insight regarding the appropriateness of the ICPC solution can be obtained by using

21 results from the numerical simulation to examine the spatial distribution of temperature radially

22 outward from the centerline of the heater probe into the medium surrounding it. We show results

23 for the case where the medium is water (Fig. 10). The labels on the curves denote the time in 
1 seconds from the onset of heating. The temperature of the heater probe increases rapidly with

2 time during the interval $0<t \leq t_{0}$, then gradually decreases after the current to the heater probe is

3 switched off. Of interest here is the fact that the portion of the heater probe made of stainless

4 steel remains nearly isothermal for all times. This portion of the heater probe therefore behaves

5 very much like a perfect conductor. The epoxy-filled portion of the heater probe also behaves

6 like a perfect conductor for times greater than $t_{0}$. Only during the heating interval does the

7 epoxy-filled portion of the probe exhibit behavior inconsistent with that of a perfect conductor.

8 Clearly, there appears to be good reason for the fact that the ICPC solution provides an excellent

9 description of the temperature of the temperature probe.

10 Returning to the results of Fig. 8, we now reconsider the fact that the ICPC solution

11 overestimates the temperature probe temperature at relatively early times and underestimates it at

12 relatively late times. Treating the probes as perfect conductors produces this result because the

13 effect of the finite radius of the probes is overestimated, resulting in underestimation of the

14 effective distance traveled by the heat-pulse signal. This causes a leftward shift of the signal

15 received by the temperature probe. The fact that the ICPC solution initially overestimates the

16 temperature and then later underestimates it is a direct consequence of this leftward shift. Despite

17 the fact that $\beta_{0} \neq 1$ for these results (Fig. 8), the finite heat capacity of the probes has no effect on

18 the differences $V_{2}^{\mathrm{P}}(t)-T(L, 0, t)$ because the ICPC solution and the numerical model both

19 account for the finite heat capacity of the probes. Interestingly, the leftward shift is small and

20 relatively similar for all three media, falling somewhere between 0.01 and $0.015 \mathrm{~s}$, which

21 suggests the possibly of introducing a time offset correction to further improve the accuracy of

22 the ICPC solution for DPHP applications. 
2 Having established the validity of the ICPC solution, and having demonstrated its usefulness

3 for DPHP applications, we now briefly consider experimental evidence suggesting that the

4 DPHP method may result in biased thermal property and water content estimates if the finite

5 properties of the probes are not taken into account. We also examine potential limitations of the

$6 \quad$ ICPC solution for situations where $a_{0} / L>0.11$.

\section{Experimental Evidence for Probe Effects}

9 Perhaps the strongest evidence for the aforementioned probe effects is found in the results of

10 Ham and Benson (2004), who calibrated DPHP sensors (i.e., measured apparent probe spacing)

11 in media with a range of known volumetric heat capacities. Apparent spacing was determined

12 from measured values of maximum temperature rise using infinite line source theory. Their

13 results showed that apparent spacing increased significantly as the heat capacity of the

14 calibration media decreased. Furthermore, they showed that apparent spacing was greater than

15 the physical probe spacing for calibration in dry glass beads whereas apparent spacing was

16 smaller than the physical spacing for calibration in saturated glass beads and agar-immobilized

17 water. These results are entirely consistent with the differences between the maximum

18 temperature rise of the ICPC and line-source solutions (Fig. 5). The results in Fig. 5 show that

19 apparent spacing will be overestimated when $\beta_{0}>1$ (as for calibration in dry glass beads) and

20 underestimated when $\beta_{0}<1$ (as for calibration in saturated glass beads and agar-immobilized

21 water). Although the results of Ham and Benson (2004) cannot be explained entirely by the finite

22 radius and finite heat capacity of the probes, these probe effects likely contributed to the

23 observed trends. Clearly, there is a need for experimental work to determine if use of the ICPC 
1 solution might minimize the dependence of apparent spacing on the heat capacity of the

2 calibration medium, or perhaps even eliminate the need to determine apparent spacing.

3 Further evidence for probe effects can be found in the fact that many have reported

4 saturation-dependent bias in water content estimates (derived from heat capacity estimates) when

5 infinite line source theory is used for parameter estimation (Tarara and Ham, 1997; Song et al.,

6 1998; Bristow et al., 2001; Basinger et. al., 2003; Ochsner et al., 2003). The bias reported in

7 these investigations is consistent with the results of Fig. 5 in that overestimation of water content

8 was greatest at smaller water contents (i.e. for smaller values of heat capacity) when the contrast

9 between the heat capacity of the probes and that of the soil would be greatest. Clearly, there is

10 also scope for experimental work to determine if use of the ICPC solution might reduce

11 saturation-dependent bias in heat capacity and water content estimates.

\section{Potential Limitations of the Solution}

14 The validity of the ICPC solution and its appropriateness for DPHP applications were

15 established using results for the case where $a_{0} / L \approx 0.11$. It follows, of course, that the solution

16 will be valid and appropriate for applications where $a_{0} / L<0.11$, but there are potential

17 limitations for applications where $a_{0} / L>0.11$. Additional investigation is required to determine

18 the extent to which $a_{0} / L$ can be increased before the ICPC solution fails to provide a good

19 approximation of the temperature probe temperature for the case where both probes have infinite

20 conductivity. Also required is analysis to determine the extent to which $a_{0} / L$ can be increased

21 before the appropriateness of the perfect conductor assumption begins to fail.

22 Notwithstanding these potential limitations of the theory, it is natural to consider whether use

23 of the ICPC solution might make it feasible to implement the DPHP method with sensors that 
1 have probes of larger radius. This possibility certainly merits consideration, but words of caution

2 are in order. The ICPC solution accounts for the finite radius of the probes, but it does not

3 account for their finite length or for the possibility of axial conduction in the probes. These

4 issues are not generally regarded to be of major concern for sensors with probe spacing and

5 probe geometry (i.e., radius and length) similar to that of the Campbell et al. (1991) and Basinger

6 et al. (2003) sensors, but they may become significant if probe radius is increased without a

7 corresponding increase in probe length. Thus, it is not a given that the radius of the probes can be

8 increased simply by using theory that accounts for their finite radius and finite heat capacity.

$9 \quad$ Experimental work as well as additional analysis will be required.

10 Finally, we emphasize that the ICPC solution does not allow for the possibility of imperfect

11 contact between the soil and the probes. In this regard, the ICPC solution is no better than the

12 line source solution. The importance of contact resistance was not addressed in this investigation,

13 but it certainly merits further consideration. It has been shown contact resistance causes minimal

14 error in heat capacity estimates (Noborio et al., 1996; Liu and Si, 2010), but Noborio et al.

15 (1996) also showed that a 0.1-mm air gap between the soil and the probes may cause error as

16 large as $10 \%$ in estimates of thermal conductivity.

\section{SUMMARY AND CONCLUSIONS}

19 We have derived a semianalytical solution that accounts for the finite radius and finite heat

20 capacity of the probes of a DPHP sensor. The solution consists of a closed-form expression in

21 the Laplace domain that is inverted numerically using the Stehfest algorithm. Equation [35] is

22 the Laplace-domain expression for the temperature of the temperature probe for the general case

23 where the heater and temperature probes have different radius and heat capacity. This result was 
1 used to derive Eq. [40], which gives the temperature of the temperature probe for the special case

2 where both probes of the DPHP sensor have the same radius and the same heat capacity.

3 Equation [40], which we refer to as the ICPC solution, was used to investigate the effects of

4 the finite radius and finite heat capacity of the probes. An important result revealed by the

5 functional form of Eq. [40] is that the finite properties of the heater probe and the finite

6 properties of the temperature probe have equal effects on the temperature of the temperature

7 probe. We also have shown that the finite radius of the probes causes the heat-pulse signal to

8 arrive at the temperature probe slightly earlier in time. The magnitude of this time shift depends

9 primarily on the magnitude of the ratio $a_{0} / L$. For a sensor with fixed probe spacing $L$, the

10 magnitude of the time shift increases as the radius of the probes increases. The effect of the finite

11 heat capacity of the probes depends on the parameter $\beta_{0}$, which is the ratio of the heat capacity of

12 the probes and the heat capacity of the soil. Relative to the case where $\beta_{0}=1$, less energy is

13 needed to raise the temperature of the probes when $\beta_{0}<1$. This causes an increase in the

14 magnitude of the heat-pulse signal, and the signal is positively skewed so the maximum

15 temperature rise occurs earlier in time. When $\beta_{0}>1$, the magnitude of the heat-pulse signal is

16 decreased and the signal is negatively skewed so the maximum temperature rise occurs later.

17 Results obtained with the ICPC solution for a typical DPHP sensor showed that the effects of

18 the finite radius and finite heat capacity of the probes are not insignificant. This is particularly

19 true when $\beta_{0}$ is large, which occurs when measurements are made in relatively dry soil. The large

20 contrast between the heat capacity of the probes and that of the soil has a substantial effect on the

21 heat-pulse signal received by the temperature probe. Our results for a typical sensor are

22 consistent with published experimental data suggesting that the DPHP method may result in 
1 biased thermal property and water content estimates if the finite properties of the probes are not

2 taken into account.

3 Several simplifying assumptions were made in deriving the semianalytical solution: (i) that

4 the probes are perfect conductors, (ii) that the temperature probe does not alter the radial

5 symmetry of the temperature distribution around the heater probe, and (iii) that there is no

6 contact resistance at the soil-probe interfaces. The validity of the first two assumptions was

7 investigated with a finite-element model that accounted for the finite conductivity as well as the

8 finite heat capacity of the probes. The results showed that both of these assumptions are valid for

9 DPHP applications where the probes satisfy the condition that $a_{0} / L \leq 0.11$. Additional

10 investigation is required to determine the extent to which $a_{0} / L$ can be increased before these

11 assumptions become inappropriate. If the ICPC solution remains valid for larger values of $a_{0} / L$,

12 it may be feasible to implement the DPHP method with sensors that have probes of larger radius.

13 This would be desirable from the standpoint of increasing the rigidity of the probes, but it is not a

14 given that the radius of the probes can be increased by simply using theory that accounts for their

15 finite radius and finite heat capacity. Other effects (e.g., axial conduction) may become

16 significant as probe radius is increased. Experimental work as well as additional analysis will be

17 required to determine whether other effects may offset the benefit gained by accounting for the

18 finite radius and finite heat capacity of the probes.

\section{NOMENCLATURE}

$21 a_{0}$ - radius of the probes for the case where they both have the same radius (m)

$22 a_{1}$ - radius of the heater probe (m)

$23 a_{2}$ - radius of the temperature probe (m) 
$1 a_{\mathrm{e}}$ - radius of the epoxy-filled region in the probes $(\mathrm{m})$

$2 a_{\mathrm{b}}$ - radius of the semicircular domain for numerical model (m)

$3 g$-rate of heat generation per unit volume for numerical model $\left(\mathrm{W} \mathrm{m}^{-3}\right)$

$4 \quad p$ - Laplace transform variable $\left(\mathrm{s}^{-1}\right)$

$5 q^{\prime}$ - rate of heat per unit length released from the heater probe or line heat source $\left(\mathrm{W} \mathrm{m}^{-1}\right)$

$6 \quad r_{1}$ - radial coordinate for coordinate system centered on the heater probe $(\mathrm{m})$

$7 \quad r_{2}-$ radial coordinate for coordinate system centered on temperature probe $(\mathrm{m})$

$8 t$ - time $(\mathrm{s})$

$9 \quad t_{0}-$ heating duration (s)

$10 x-$ Cartesian coordinate $(\mathrm{m})$

$11 y$-Cartesian coordinate $(\mathrm{m})$

$12 C$ - volumetric heat capacity of the soil $\left(\mathrm{J} \mathrm{m}^{-3} \mathrm{~K}^{-1}\right)$

$13 C_{0}$ - volumetric heat capacity of the probes for the case where they both have the same heat

$14 \quad$ capacity $\left(\mathrm{J} \mathrm{m}^{-3} \mathrm{~K}^{-1}\right)$

$15 C_{1}$ - volumetric heat capacity of the heater probe $\left(\mathrm{J} \mathrm{m}^{-3} \mathrm{~K}^{-1}\right)$

$16 C_{2}$ - volumetric heat capacity of the temperature probe $\left(\mathrm{J} \mathrm{m}^{-3} \mathrm{~K}^{-1}\right)$

$17 C_{\mathrm{e}}$ - volumetric heat capacity of the thermally-conductivity epoxy $\left(\mathrm{J} \mathrm{m}^{-3} \mathrm{~K}^{-1}\right)$

$18 C_{\mathrm{ss}}-$ volumetric heat capacity of the stainless-steel tubing $\left(\mathrm{J} \mathrm{m}^{-3} \mathrm{~K}^{-1}\right)$

$19 I_{m}-$ modified Bessel function of the first kind of order $m$

$20 K_{n}-$ modified Bessel function of the second kind of order $n$

$21 L$-distance between the centerlines of the heater and temperature probes $(\mathrm{m})$

$22 T$-temperature of the soil and probes in the numerical simulations $(\mathrm{K})$

$23 \quad V_{1}$ - temperature of the heater probe in the semianalytical solution $(\mathrm{K})$ 
$1 \quad V_{2}-$ temperature of the temperature probe in the semianalytical solution $(\mathrm{K})$

$2 \beta_{0}$ - ratio of the volumetric heat capacity of the probes and the volumetric heat capacity of the

3 soil for the case where both probes have the same heat capacity (dimensionless)

$4 \quad \beta_{1}$ - ratio of the volumetric heat capacity of the heater probe and the volumetric heat capacity of

$5 \quad$ the soil (dimensionless)

$6 \quad \beta_{2}$-ratio of the volumetric heat capacity of the temperature probe and the volumetric heat

$7 \quad$ capacity of the soil (dimensionless)

$8 \theta$ - angular coordinate for coordinate system centered on temperature probe (radians)

$9 \phi$-arbitrary heating function

$10 \kappa-$ thermal diffusivity of the soil $\left(\mathrm{m}^{2} \mathrm{~s}^{-1}\right)$

$11 \lambda$-thermal conductivity of the soil $\left(\mathrm{W} \mathrm{m}^{-1} \mathrm{~K}^{-1}\right)$

$12 v$-temperature of the soil in the semianalytical solution (K)

\section{APPENDIX A}

15 The line-source solution of Bristow et al. (1994) is widely used for DPHP applications. Here

16 we show that their solution is a special case of a general line-source solution. We also give the

17 Laplace transform of that solution. The transform of the general line-source solution is of interest

18 because it appears in the derivation of the semianalytical solution.

19 Consider a line source of infinite length that releases heat into an infinite medium initially at

20 zero temperature. The line source is located at $(x, y)=(0,0)$, so the radial distance from the line

21 source in the $x-y$ plane is $r_{1}$, where $r_{1}^{2}=x^{2}+y^{2}$. If heat is released at constant rate during the

22 time interval $0<t \leq t_{0}$, the solution of the heat equation is (de Vries, 1952) 


$$
\begin{gathered}
v\left(r_{1}, t\right)=-\frac{q^{\prime}}{4 \pi \lambda} \operatorname{Ei}\left(-\frac{r_{1}^{2}}{4 \kappa t}\right) ; \quad 0<t \leq t_{0} \\
v\left(r_{1}, t\right)=\frac{q^{\prime}}{4 \pi \lambda}\left\{\operatorname{Ei}\left[-\frac{r_{1}^{2}}{4 \kappa\left(t-t_{0}\right)}\right]-\operatorname{Ei}\left(-\frac{r_{1}^{2}}{4 \kappa t}\right)\right\} ; \quad t>t_{0}
\end{gathered}
$$

2 where $q^{\prime}$ is the rate per unit length at which heat is released, $t_{0}$ is the heating duration, and $-\operatorname{Ei}(-$

$3 x$ ) is the exponential integral of argument $x$. This is the solution employed in the DPHP method

4 of Bristow et al. (1994).

5 It is easily shown that Eq. [A1] is a special case of the general solution (Carslaw and Jaeger,

$6 \quad 1959$, p. 261)

$$
v\left(r_{1}, t\right)=\frac{1}{4 \pi \lambda} \int_{0}^{t} \phi\left(t^{\prime}\right) \exp \left[-\frac{r_{1}^{2}}{4 \kappa\left(t-t^{\prime}\right)}\right] \frac{d t^{\prime}}{t-t^{\prime}}
$$

8 For pulsed heating, the general line-source solution yields Eq. [A1] by using the form for $\phi(t)$

9 given in Eq. [38]. By making use of Duhamel's theorem (Carslaw and Jaeger, 1995, p. 301), it

10 can be shown that the Laplace transform of the general line-source solution is

$$
\hat{v}\left(r_{1}, p\right)=\frac{\hat{\phi}(p)}{2 \pi \lambda} K_{0}\left(\mu r_{1}\right)
$$

12 In the body of the text, we refer to Eq. [A3] in discussing the form of Eq. [18]. We also use Eq.

13 [A3] with $r_{1}=L$ in discussing the form of Eq. [35].

\section{APPENDIX B}

16 The Stehfest algorithm for inverting Eq. [40a] can be written in the form

$$
V_{2}^{\mathrm{C}}(t) \approx \frac{q^{\prime}}{2 \pi \lambda} \sum_{i=1}^{N} \frac{\omega_{i}}{i} \frac{K_{0}\left(\mu_{i} L\right)}{\left\{\mu_{i} a_{0}\left[K_{1}\left(\mu_{i} a_{0}\right)+\left(\mu_{i} a_{0} \beta_{0} / 2\right) K_{0}\left(\mu_{i} a_{0}\right)\right]\right\}^{2}}
$$

18 where 


$$
\mu_{i}=\sqrt{\frac{\ln (2)}{\kappa t} i} ; \quad i=1,2, \ldots, N
$$

2 All inversion calculations were performed by evaluating Eq. [B1] with $N=16$ and the Stehfest

3 weighting coefficients, $\omega_{i}$, given in Table B1. Upon replacing $t$ with $t-t_{0}$ in Eq. [B1] and [B2],

4 the same algorithm can be used to obtain values of $V_{2}^{\mathrm{C}}\left(t-t_{0}\right)$ for times greater than $t_{0}$. Inasmuch

5 as Eq. [B1] is a function of time, it must be evaluated at each time $t$ for which a value of $V_{2}^{\mathrm{C}}(t)$ is

6 desired. It must also be evaluated at each time $t-t_{0}$ for which a value of $V_{2}^{\mathrm{C}}\left(t-t_{0}\right)$ is desired.

7 The values of $V_{2}^{\mathrm{C}}(t)$ and $V_{2}^{\mathrm{C}}\left(t-t_{0}\right)$ obtained in this way were used to evaluate Eq. [40b].

8 We used $N=16$ for our calculations because (i) MATLAB's double precision floating-point

9 arithmetic yields results with approximately 16 significant decimal digits, and (ii) the function

10 BESSELK evaluates the Bessel functions $K_{0}(\cdot)$ and $K_{1}(\cdot)$ to an accuracy of about 16 decimal

11 places. Optimal results are generally obtained when $N$ is about equal to the number of significant

12 decimal digits used in the calculations (Knight and Raiche, 1982).

\section{ACKNOWLEDGMENTS}

15 This material is based on work supported by the National Science Foundation under Grant 16 No. ECS-0410055.

\section{REFERENCES}

19 Basinger, J.M., G.J. Kluitenberg, J.M. Ham, J.M. Frank, P.L. Barnes, and M.B. Kirkham. 2003.

20 Laboratory evaluation of the dual-probe heat-pulse method for measuring soil water content.

$21 \quad$ Vadose Zone J. 2:389-399. 
1 Blackwell, J.H. 1954. A transient-flow method for determination of thermal constants of

2 insulating materials in bulk: Part I. Theory. J. Appl. Phys. 25:137-144.

3 Bristow, K.L., G.J. Kluitenberg, C.J. Goding, and T.S. Fitzgerald. 2001. A small multi-needle

4 probe for measuring soil thermal properties, water content and electrical conductivity.

5 Comput. Electron. Agric. 31:265-280.

6 Bristow, K.L., G.J. Kluitenberg, and R. Horton. 1994. Measurement of soil thermal properties

7 with a dual-probe heat-pulse technique. Soil Sci. Soc. Am. J. 58:1288-1294.

8 Campbell, G.S., C. Calissendorff, and J.H. Williams. 1991. Probe for measuring soil specific

9 heat using a heat-pulse method. Soil Sci. Soc. Am. J. 55:291-293.

10 Carslaw, H.S., and J.C. Jaeger. 1959. Conduction of heat in solids. 2nd ed. Clarendon Press, 11 Oxford, UK.

12 de Vries, D.A. 1952. A nonstationary method for determining thermal conductivity of soil in 13 situ. Soil Sci. 73:83-89.

14 Guaraglia, D.O., and J.L. Pousa. 1999. An electrical model of heat flow in soil. Soil Sci. Soc. 15 Am. J. 63:457-463.

16 Ham, J.M., and E.J. Benson. 2004. On the construction and calibration of dual-probe heat 17 capacity sensors. Soil Sci. Soc. Am. J. 68:1185-1190.

18 Hopmans, J.W., J. Simunek, and K.L. Bristow. 2002. Indirect estimation of soil thermal

19 properties and water flux using heat pulse probe measurements: Geometry and dispersion 20 effects. Water Resour. Res. 38, doi:10.1029/2000WR000071.

21 Incropera, F.P., and D.P. De Witt. 1996. Fundamentals of heat and mass transfer. 4th ed. John 22 Wiley \& Sons, New York. 
1 Jaeger, J.C. 1956. Conduction of heat in an infinite region bounded internally by a circular

2 cylinder of a perfect conductor. Aust. J. Phys. 9:167-179.

3 Kamai, T., G.J. Kluitenberg, and J.W. Hopmans. 2009. Design and numerical analysis of a

4 button heat pulse probe for soil water content measurement. Vadose Zone J. 8:167-173.

5 Knight, J.H., W. Jin, and G.J. Kluitenberg. 2007. Sensitivity of the dual-probe heat-pulse method

6 to spatial variations in heat capacity and water content. Vadose Zone J. 6:746-758.

7 Knight, J.H., and A.P. Raiche. 1982. Transient electromagnetic calculations using the Gaver-

8 Stehfest inverse Laplace transform method. Geophysics 47:47-50.

9 Liu, G., and B.C. Si. 2010. Errors analysis of heat pulse probe methods: Experiments and

10 simulations. Soil Sci. Soc. Am. J. 74:797-803.

11 Noborio, K., K.J. McInnes, and J.L. Heilman. 1996. Measurements of soil water content, heat 12 capacity, and thermal conductivity with a single TDR probe. Soil Sci. 161:22-28.

13 Novakowski, K.S. 1989. Analysis of pulse interference tests. Water Resour. Res. 11:2377-2387.

14 Nusier, O.K., and N.H. Abu-Hamdeh. 2003. Laboratory techniques to evaluate thermal

15 conductivity for some soils. Heat Mass Transfer 39:119-123.

16 Ochsner, T.E., R. Horton, and T. Ren. 2003. Use of the dual-probe heat-pulse technique to

17 monitor soil water content in the vadose zone. Vadose Zone J. 2:572-579.

18 Ogbe, D.O., and W.E. Brigham. 1984. A model for interference testing with wellbore and skin

19 effects at both wells. Paper SPE 13253 presented at the 59th Annual Technical Conference

20 and Exhibition, Houston, TX, Sept. 16-19, 1984. Society of Petroleum Engineers.

21 Olver, F.W.J. 1965. Bessel functions of integer order. p. 355-433. In M. Abramowitz and I.A.

22 Stegun (ed.) Handbook of mathematical functions with formulas, graphs, and mathematical 23 tables. Dover, New York. 
1 Ren, T., G.J. Kluitenberg, and R. Horton. 2000. Determining soil water flux and pore water

2 velocity by a heat pulse technique. Soil Sci. Soc. Am. J. 64:552-560.

3 Ren, T., K. Noborio, and R. Horton. 1999. Measuring soil water content, electrical conductivity,

4 and thermal properties with a thermo-time domain reflectometry probe. Soil Sci. Soc. Am. J. $5 \quad 63: 450-457$.

6 Song, Y., J.M. Ham, M.B. Kirkham, and G.J. Kluitenberg. 1998. Measuring soil water content

7 under turfgrass using the dual-probe heat-pulse technique. J. Am. Soc. Hortic. Sci. 123:9378941.

9 Stehfest, H. 1970a. Algorithm 368: Numerical inversion of Laplace transforms [D5]. Commun. $10 \quad$ ACM 13:47-49.

11 Stehfest, H. 1970b. Remark on Algorithm 368 [D5]: Numerical inversion of the Laplace 12 transforms. Commun. ACM 13:624.

13 Tarara, J.M., and J.M. Ham. 1997. Measuring soil water content in the laboratory and field with 14 dual-probe heat-capacity sensors. Agron. J. 89:535-542.

15 Tongpenyai. Y., and R. Raghavan. 1981. The effect of wellbore storage and skin on interference 16 test data. J. Pet. Technol. 33:151-160.

\section{FIGURE CAPTIONS}

19 Fig. 1. Coordinate systems used in deriving the semianalytical solution. The heater probe has 20 radius $a_{1}$, the temperature probe has radius $a_{2}$, and the centerlines of the probes are a distance $21 L$ apart. The polar coordinate system centered on the heater probe at $(x, y)=(0,0)$ has radial 22 coordinate $r_{1}$, where $r_{1}^{2}=x^{2}+y^{2}$. The polar coordinate system centered on the temperature 
probe at $(x, y)=(L, 0)$ has coordinates $r_{2}$ and $\theta$ that satisfy the conditions $r_{2}^{2}=(x-L)^{2}+y^{2}$ and $(x-L, y)=\left(r_{2} \cos \theta, r_{2} \sin \theta\right)$.

Fig. 2. Temperature (or change in temperature) of the temperature probe as a function of time. Results for the ICPC solution are from Eq. [40] with $\beta_{0}=1$ and dimensionless probe sizes $a_{0} / L$ of $0.05,0.10$, and 0.15 . Results for the line-source solution are from Eq. [A1] with $r_{1}=$ $L$. The ICPC solution is identical to the line-source solution for the limiting case where $a_{0} \rightarrow$ 0 . Both solutions were evaluated using $q^{\prime}=100 \mathrm{~W} \mathrm{~m}^{-1}, t_{0}=8 \mathrm{~s}, L=6.0 \times 10^{-3} \mathrm{~m}, C=2.0 \mathrm{MJ}$ $\mathrm{m}^{-3} \mathrm{~K}^{-1}$, and $\lambda=0.5 \mathrm{~W} \mathrm{~m}^{-1} \mathrm{~K}^{-1}$.

Fig. 3. Temperature (or change in temperature) of the temperature probe as a function of time. Results for the ICPC solution are from Eq. [40] with $a_{0} / L=0.1$ and $\beta_{0}$ values of 0.5, 1.0, and 2.0. Results for the line-source solution are from Eq. [A1] with $r_{1}=L$. The ICPC solution is identical to the line-source solution for the limiting case where $a_{0} \rightarrow 0$. Both solutions were evaluated using $q^{\prime}=100 \mathrm{~W} \mathrm{~m}^{-1}, t_{0}=8 \mathrm{~s}, L=6.0 \times 10^{-3} \mathrm{~m}, C=2.0 \mathrm{MJ} \mathrm{m}^{-3} \mathrm{~K}^{-1}$, and $\lambda=$ $0.5 \mathrm{~W} \mathrm{~m}^{-1} \mathrm{~K}^{-1}$.

Fig. 4. Cross-section of the heater probe (left) and temperature probe (right) of the dual-probe heat-pulse sensor of Basinger et al. (2003). The cross-section is for a plane normal to the axes of the probes that coincides with the location of the thermistor. The heating element in the heater probe consists of four strands of resistance wire. The portions of the probes filled with thermally conductive epoxy are shown in white.

Fig. 5. Temperature (or change in temperature) of the temperature probe as a function of time for dry sand $\left(\beta_{0}=2.58\right)$, wet sand $\left(\beta_{0}=0.93\right)$, and water $\left(\beta_{0}=0.68\right)$. Results for the ICPC solution are from Eq. [40] with $a_{0} / L \approx 0.11$. Results for the line-source solution are from Eq. 
1 [A1] with $r_{1}=L$. Both solutions were evaluated using media thermal properties from Table 1

2 and the parameter values given in Table 2.

3 Fig. 6. Mesh of triangular elements used for the numerical model. (A) Mesh for the heater and

4 temperature probes and a portion of the soil surrounding them. The $x$ axis coincides with the

5 horizontal line that passes through the center of the probes. (B) Expanded view of the mesh

6 for the temperature probe and a portion of the soil surrounding it. The small circle identifies

7 the node at $(x, y)=(L, 0)$. The temperature at this node was taken to represent the

8 temperature recorded by the thermistor.

9 Fig. 7. Difference between the temperature of the temperature probe determined using the ICPC

10 solution, $V_{2}^{\mathrm{P}}(t)$, and the temperature of the temperature probe determined using the

11 numerical model, $T(L, 0, t)$. The difference $V_{2}^{\mathrm{P}}(t)-T(L, 0, t)$ is shown as a function of time

12 for dry sand, wet sand, and water. Results for the ICPC solution are from Eq. [40]. Results

13 from the numerical model are for the case where both probes were assigned an arbitrarily

14 large value of conductivity so they were effectively perfect conductors. The ICPC solution

15 and the numerical model were both evaluated using media thermal properties from Table 1

16 and the parameter values given in Table 2. Values for $C_{\mathrm{e}}$ and $C_{\mathrm{ss}}$ in the numerical model were

17 from Table 1.

18 Fig. 8. Difference between the temperature of the temperature probe determined using the ICPC

19 solution, $V_{2}^{\mathrm{P}}(t)$, and the temperature of the temperature probe determined using the

20 numerical model, $T(L, 0, t)$. The difference $V_{2}^{\mathrm{P}}(t)-T(L, 0, t)$ is shown as a function of time

21 for dry sand, wet sand, and water. Results for the ICPC solution are from Eq. [40]. Results

22 from the numerical model are for the case where the probes had finite conductivity as well as

23 finite heat capacity. Both solutions were evaluated using media thermal properties from 
Table 1 and the parameter values given in Table 2. For the numerical model, values from

2 Table 1 were used for the thermal properties of the probe materials.

3 Fig. 9. Difference between the temperature of the temperature probe determined using the line-

4 source solution, $\mathrm{v}(L, t)$, and the temperature of the temperature probe determined using the

5 numerical model, $T(L, 0, t)$. The difference $v(L, t)-T(L, 0, t)$ is shown as a function of time

6 for dry sand, wet sand, and water. Results for the line-source solution are from Eq. [A1] with

$7 \quad r_{1}=L$. Results from the numerical model are for the case where the probes had finite

8 conductivity as well as finite heat capacity. Both solutions were evaluated using media

9 thermal properties from Table 1 and the parameter values given in Table 2. For the numerical

10 model, values from Table 1 were used for the thermal properties of the probe materials.

11 Fig. 10. Spatial distribution of temperature radially outwards from the centerline of the heater

12 probe into water. The labels on the curves denote the time in seconds from the onset of

13 heating. The open triangle shows the location of the interface between the epoxy and

14 stainless steel at $r_{1}=a_{\mathrm{e}}$. The filled triangle shows the location of the interface between the

15 stainless steel and water at $r_{1}=a_{0}$. Results from the numerical model are for the case where

16 the probes had finite conductivity as well as finite heat capacity. The model was evaluated

17 using media thermal properties from Table 1 and the parameter values given in Table 2.

18 Values from Table 1 were used for the thermal properties of the probe materials. 
Table 1 . Volumetric heat capacity $(C)$, thermal conductivity $(\lambda)$, and thermal diffusivity ( $\kappa)$ of the materials used in this investigation. The relationship $\kappa=\lambda / C$ was used to calculate diffusivity values.

\begin{tabular}{cccc}
\hline Material & $C$ & $\lambda$ & $\kappa$ \\
\hline & $\mathrm{MJ} \mathrm{m}^{-3} \mathrm{~K}^{-1}$ & $\mathrm{~W} \mathrm{~m}^{-1} \mathrm{~K}^{-1}$ & $\mathrm{~m}^{2} \mathrm{~s}^{-1}$ \\
Type 304 stainless steel & $3.77 \dagger$ & $14.9 \dagger$ & $3.95 \times 10^{-6}$ \\
Omegabond 101 epoxy & $1.64 \ddagger$ & $1.04 \ddagger$ & $6.34 \times 10^{-7}$ \\
Air-dried Clayton sand & $1.1 \S$ & $0.3 \S$ & $2.73 \times 10^{-7}$ \\
Saturated Hanlon sand & $3.07 \uparrow$ & 1.959 & $6.35 \times 10^{-7}$ \\
Water & 4.18 & 0.60 & $1.44 \times 10^{-7}$ \\
\hline
\end{tabular}

$\dagger$ Values from Table A.1 of Incropera and De Witt (1996).

\$Values from Table 1 of Kamai et al. (2009).

$\S$ Values adapted from Table 2 of Bristow et al. (1994).

II Values from Table 2 of Ren et al. (2000).

Table 2. Parameter values corresponding to the DPHP sensor of Basinger et al. (2003).

\begin{tabular}{ccc}
\hline Parameter & Value & Units \\
\hline$a_{\mathrm{e}}$ & $4.19 \times 10^{-4}$ & $\mathrm{~m}$ \\
$a_{0}$ & $6.35 \times 10^{-4}$ & $\mathrm{~m}$ \\
$L$ & $6.00 \times 10^{-3}$ & $\mathrm{~m}$ \\
$C_{0}$ & 2.84 & $\mathrm{MJ} \mathrm{m}^{-3} \mathrm{~K}^{-1}$ \\
$q^{\prime}$ & 100 & $\mathrm{~W} \mathrm{~m}^{-1}$ \\
$t_{0}$ & 8 & $\mathrm{~s}$ \\
\hline
\end{tabular}


Table 3. Estimates of volumetric heat capacity $(C)$, thermal conductivity $(\lambda)$, and thermal diffusivity $(\kappa)$ determined by using the method of Bristow et al. (1994) to fit the line-source solution to the curves for the ICPC solution in Fig. 5. The values in parentheses give the relative errors (\%) in the estimated thermal properties (i.e. the errors in estimated thermal properties expressed as a percent of the original values in Table 1).

\begin{tabular}{cccc}
\hline Material & $C$ & $\lambda$ & $\kappa$ \\
\hline & $\mathrm{MJ} \mathrm{m}^{-3} \mathrm{~K}^{-1}$ & $\mathrm{~W} \mathrm{~m}^{-1} \mathrm{~K}^{-1}$ & $\mathrm{~m}^{2} \mathrm{~s}^{-1}$ \\
Air-dried Clayton sand & $1.17(6.4)$ & $0.28(-7.7)$ & $2.36 \times 10^{-7}(-13.5)$ \\
Saturated Hanlon sand & $3.06(-0.3)$ & $2.01(3.0)$ & $6.57 \times 10^{-7}(3.4)$ \\
Water & $4.12(-1.4)$ & $0.63(5.0)$ & $1.53 \times 10^{-7}(6.6)$ \\
\hline
\end{tabular}

Table B1. Weighting coefficients $\omega_{i}$ for the algorithm of Stehfest (1970a,b) for the case where $N=16$.

\begin{tabular}{cc}
\hline$i$ & $\omega_{i}$ \\
\hline 1 & $-3.96825396825396825 \times 10^{-4}$ \\
2 & 2.13373015873016 \\
3 & -551.01666666666667 \\
4 & 33500.161111111111 \\
5 & -812665.11111111111111 \\
6 & 10076183.766666667 \\
7 & -73241382.977777778 \\
8 & 339059632.073016 \\
9 & -1052539536.278571 \\
10 & 2259013328.5833333 \\
11 & -3399701984.4333333 \\
12 & 3582450461.7 \\
13 & -2591494081.3666667 \\
14 & 1227049828.7666667 \\
15 & -342734555.42857143 \\
16 & 42841819.428571428 \\
\hline
\end{tabular}


Figure 1

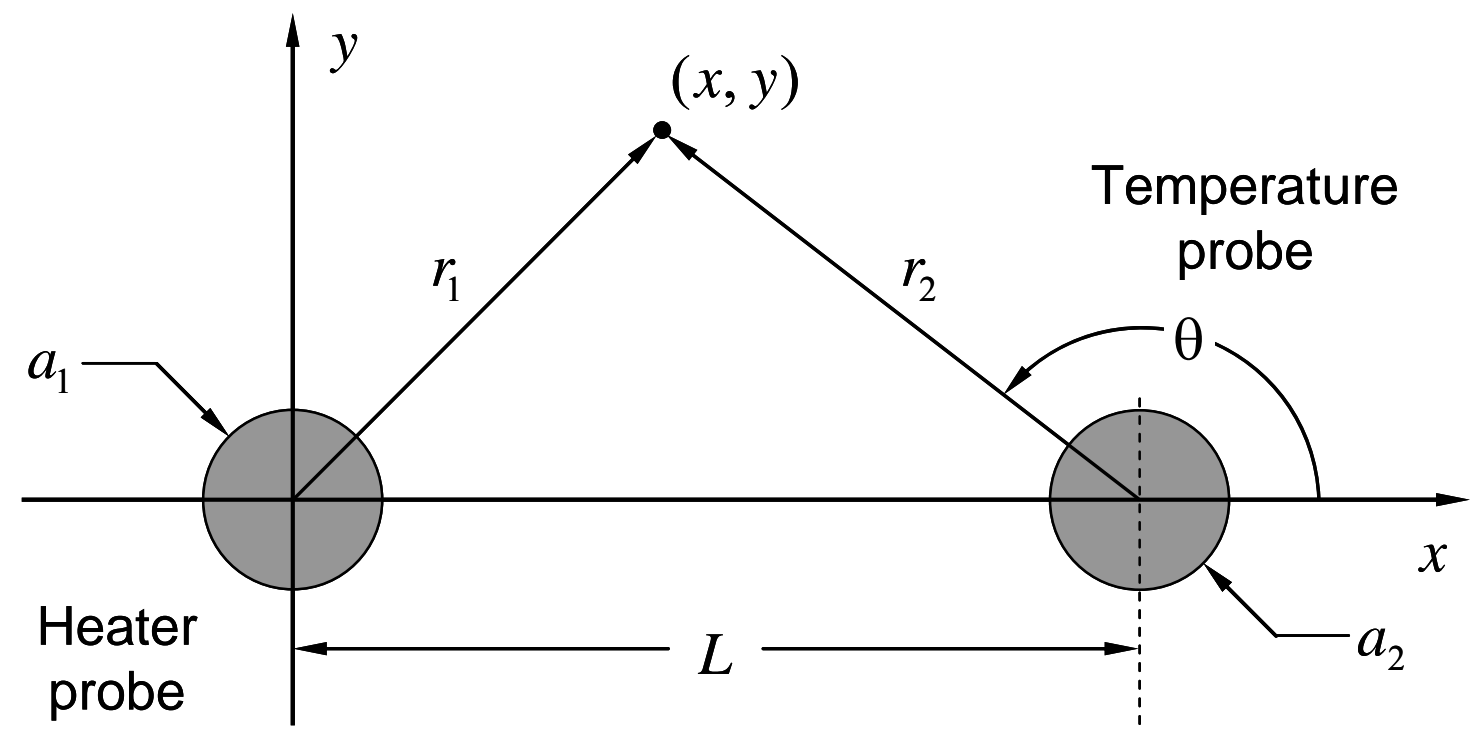


Figure 2

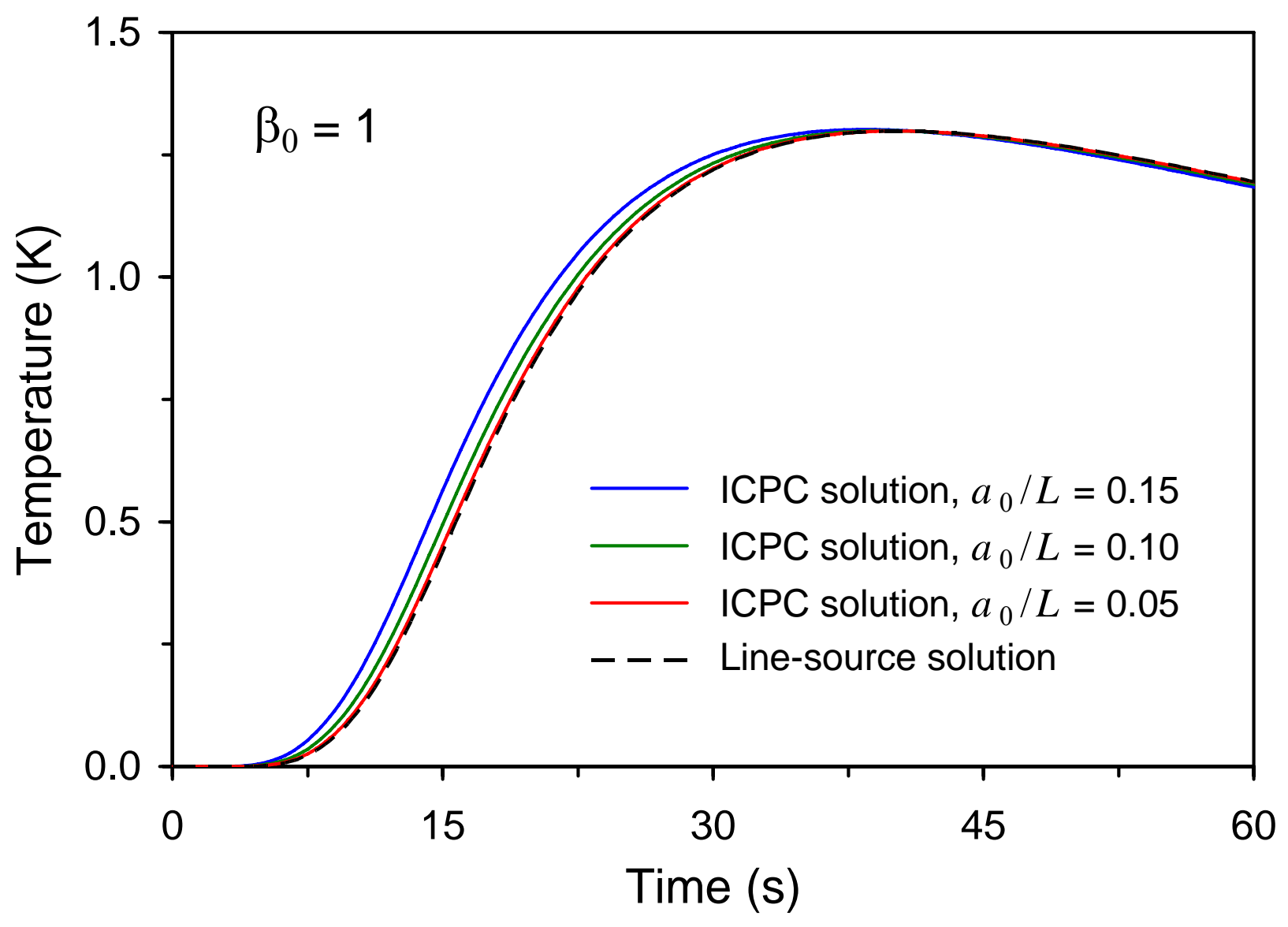


Figure 3

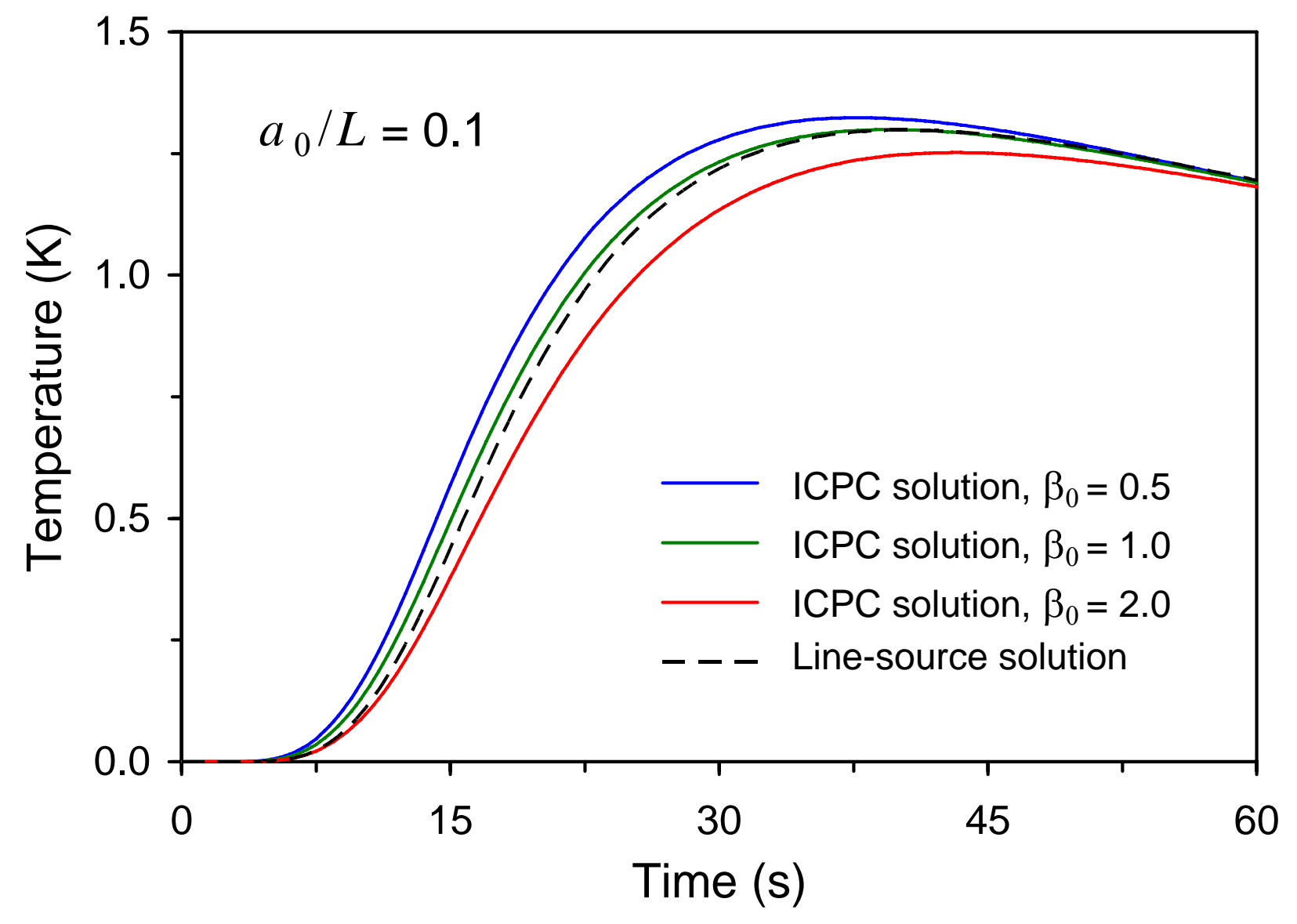


Figure 4

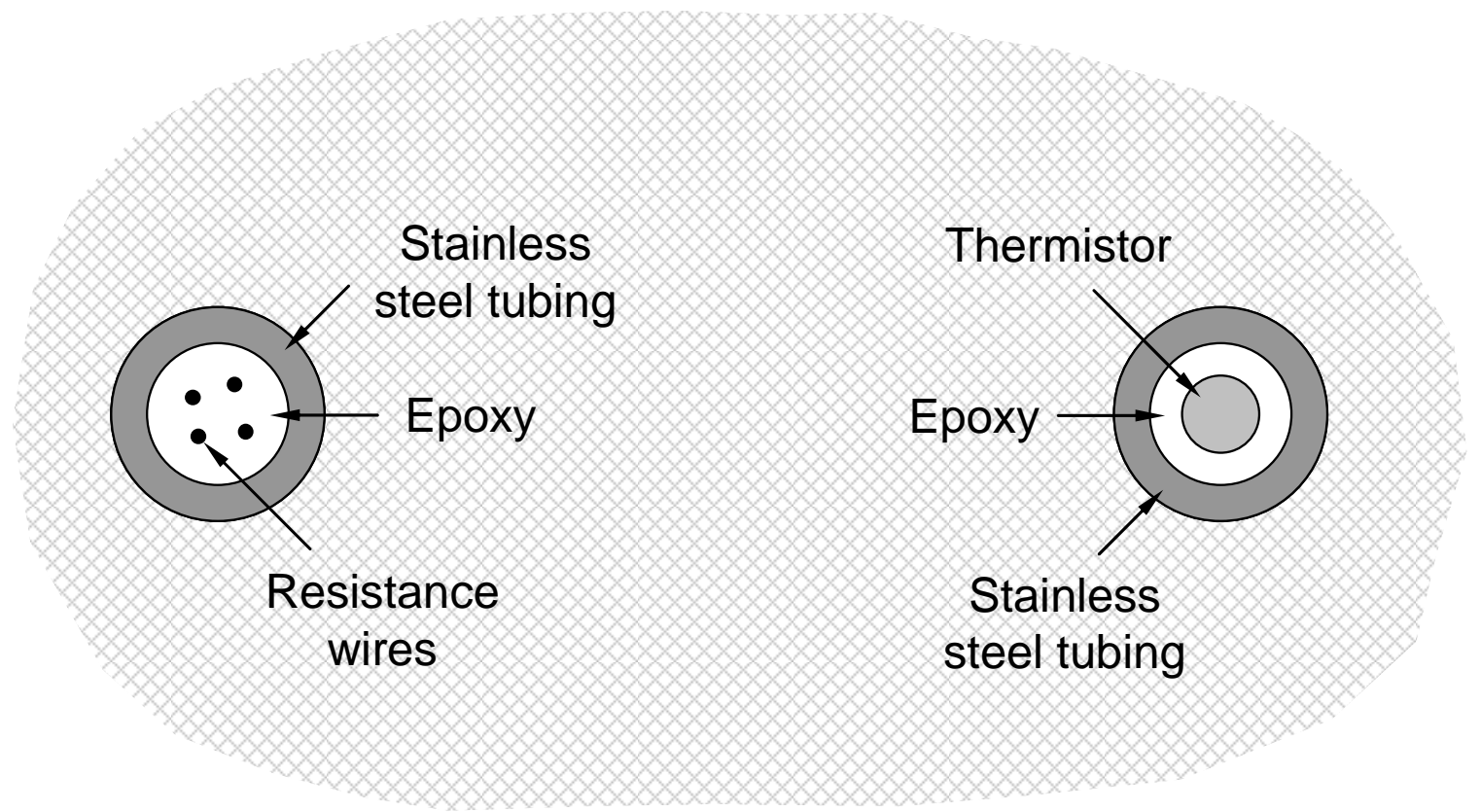




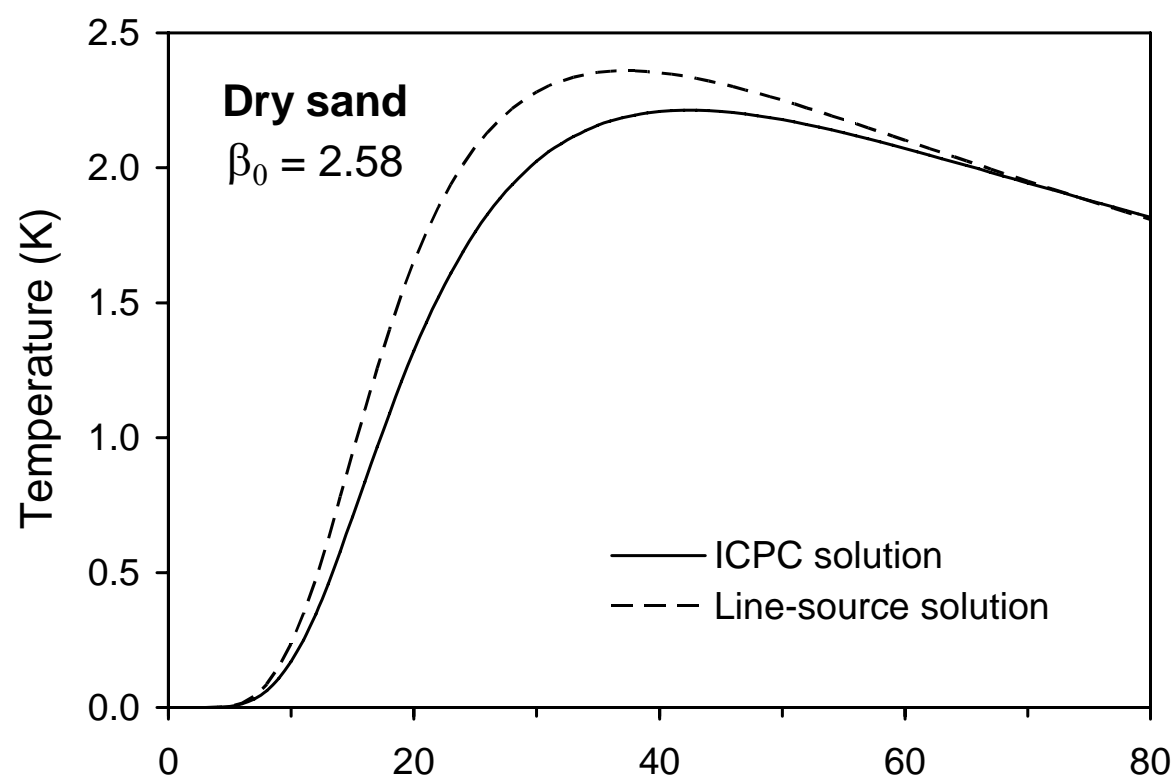

Figure 5
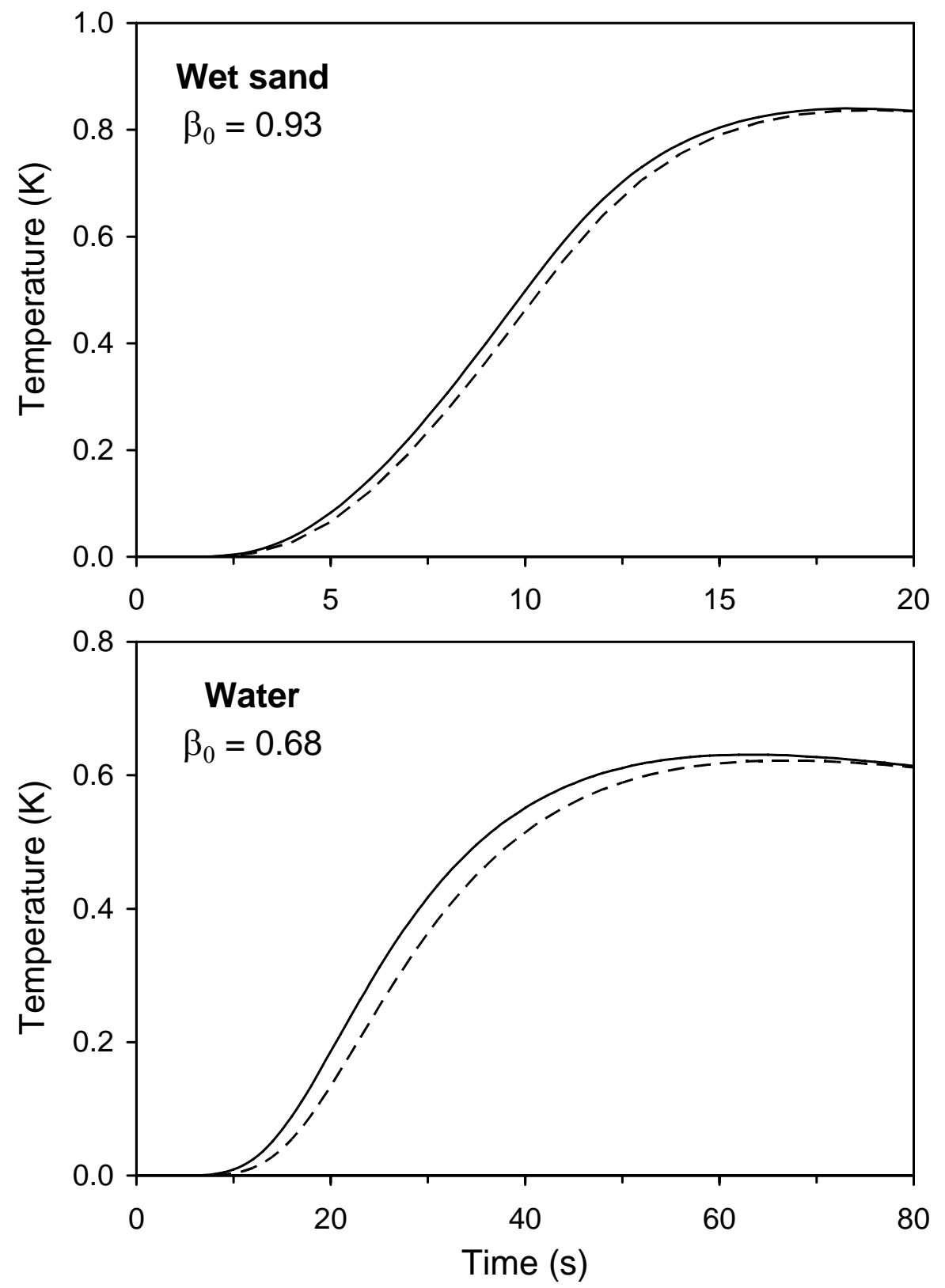
Figure 6a

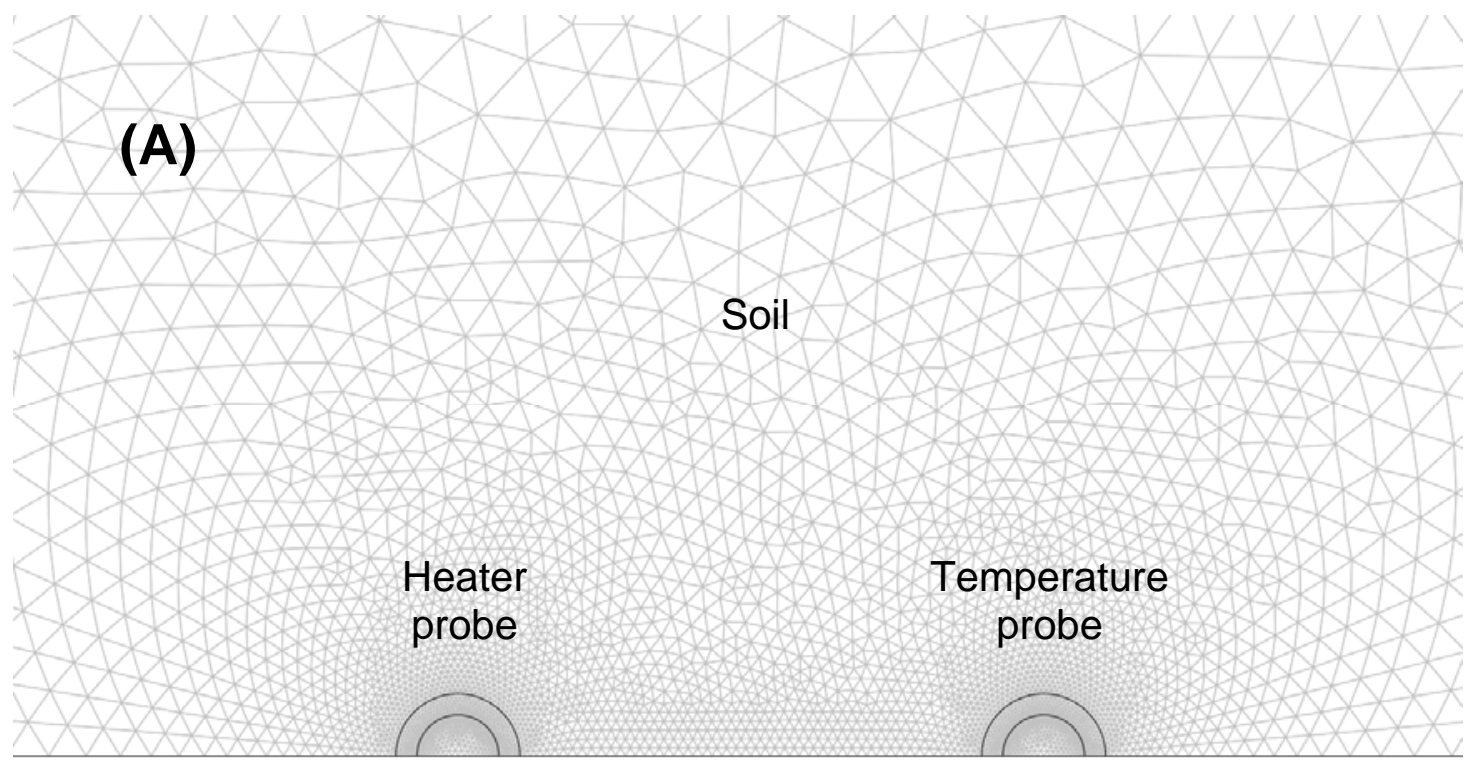

Figure $6 b$

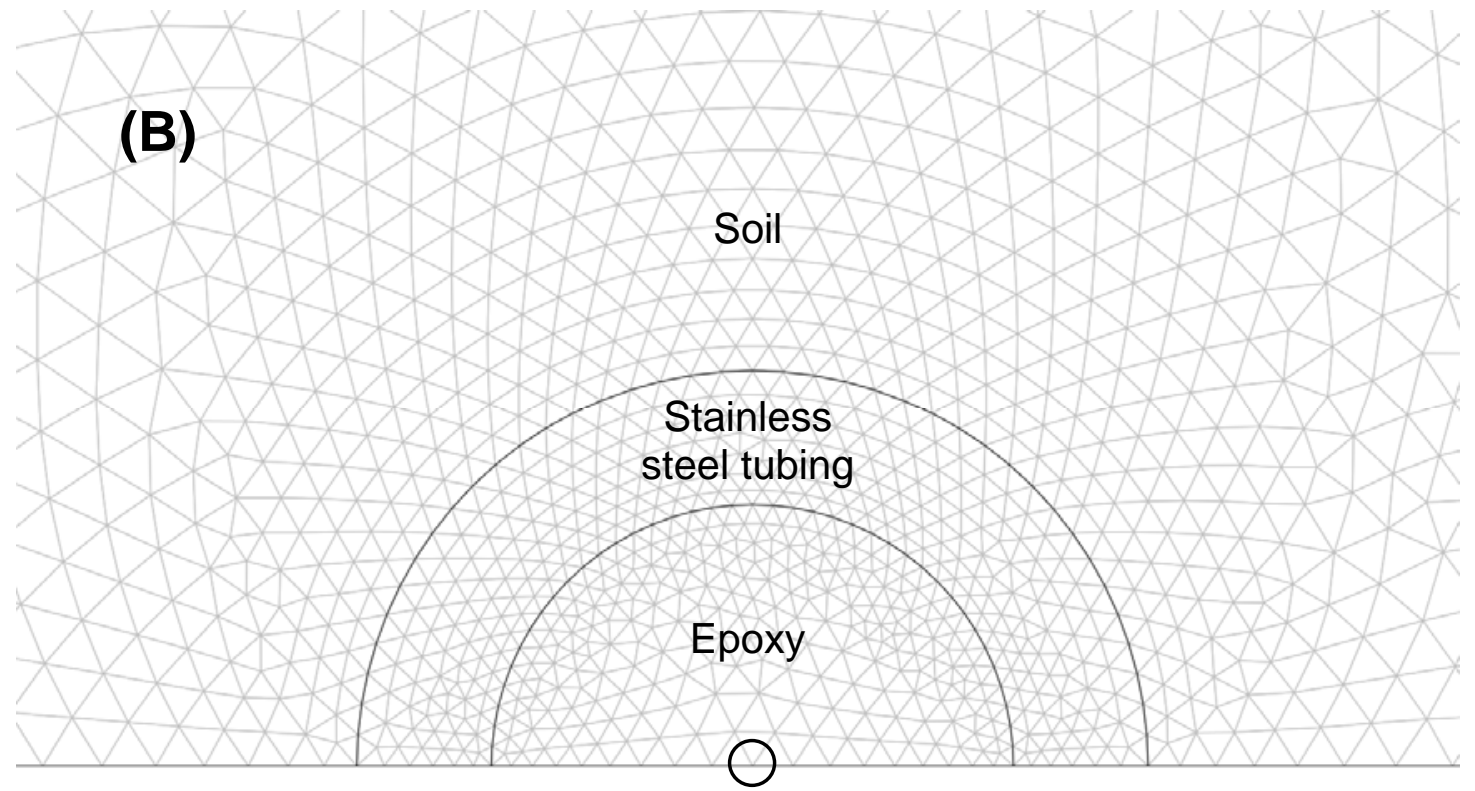


Figure 7

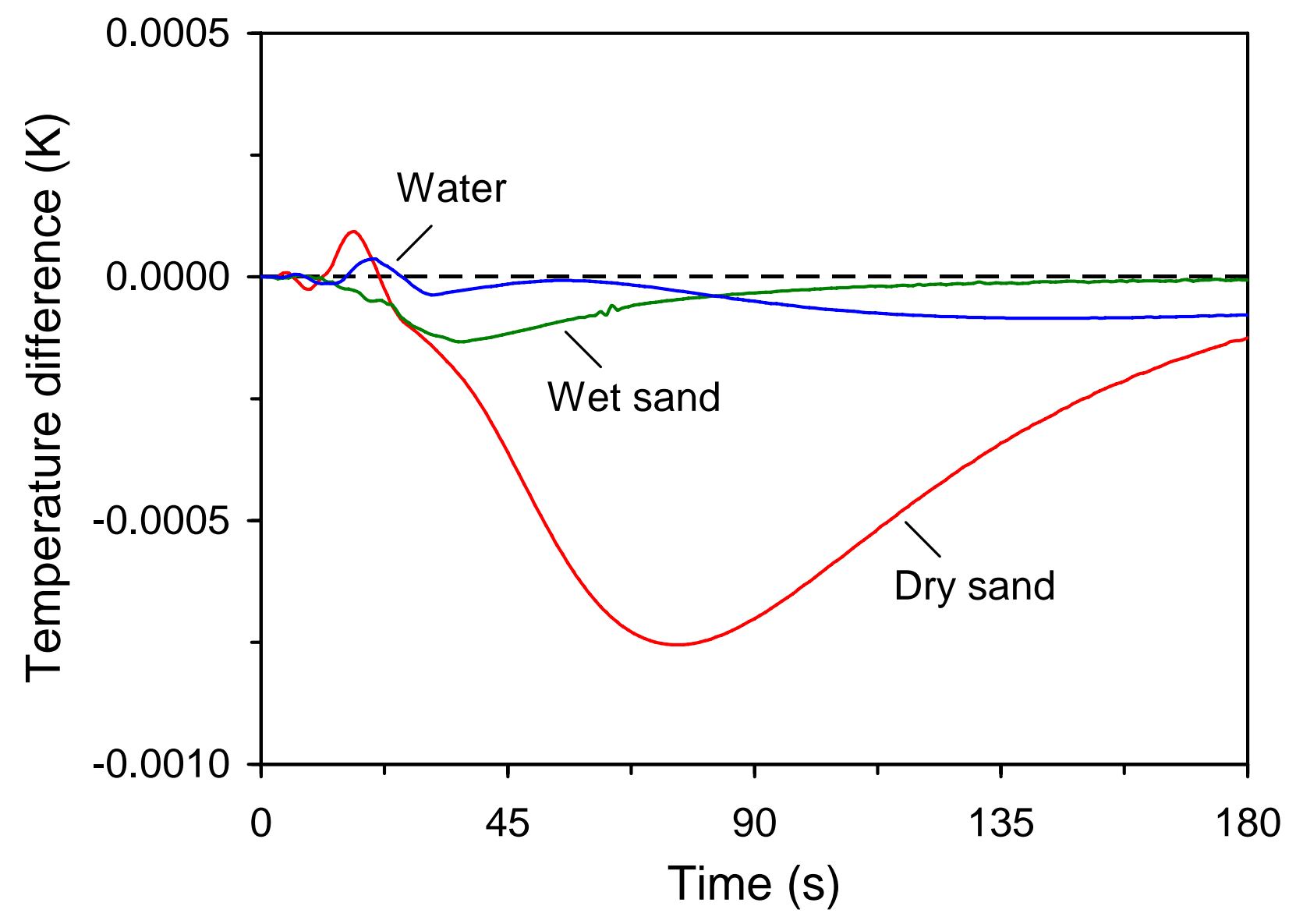


Figure 8

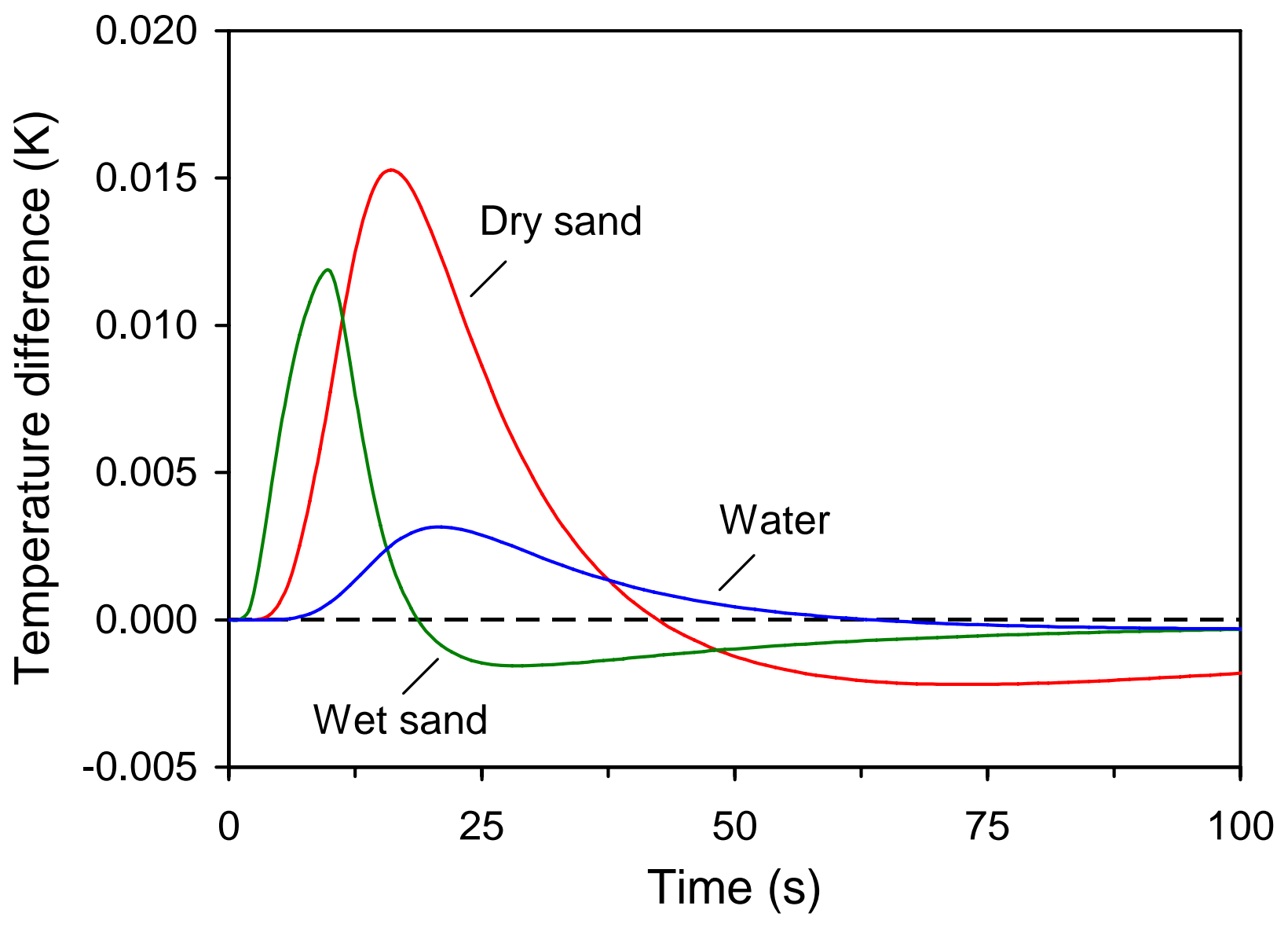


Figure 9

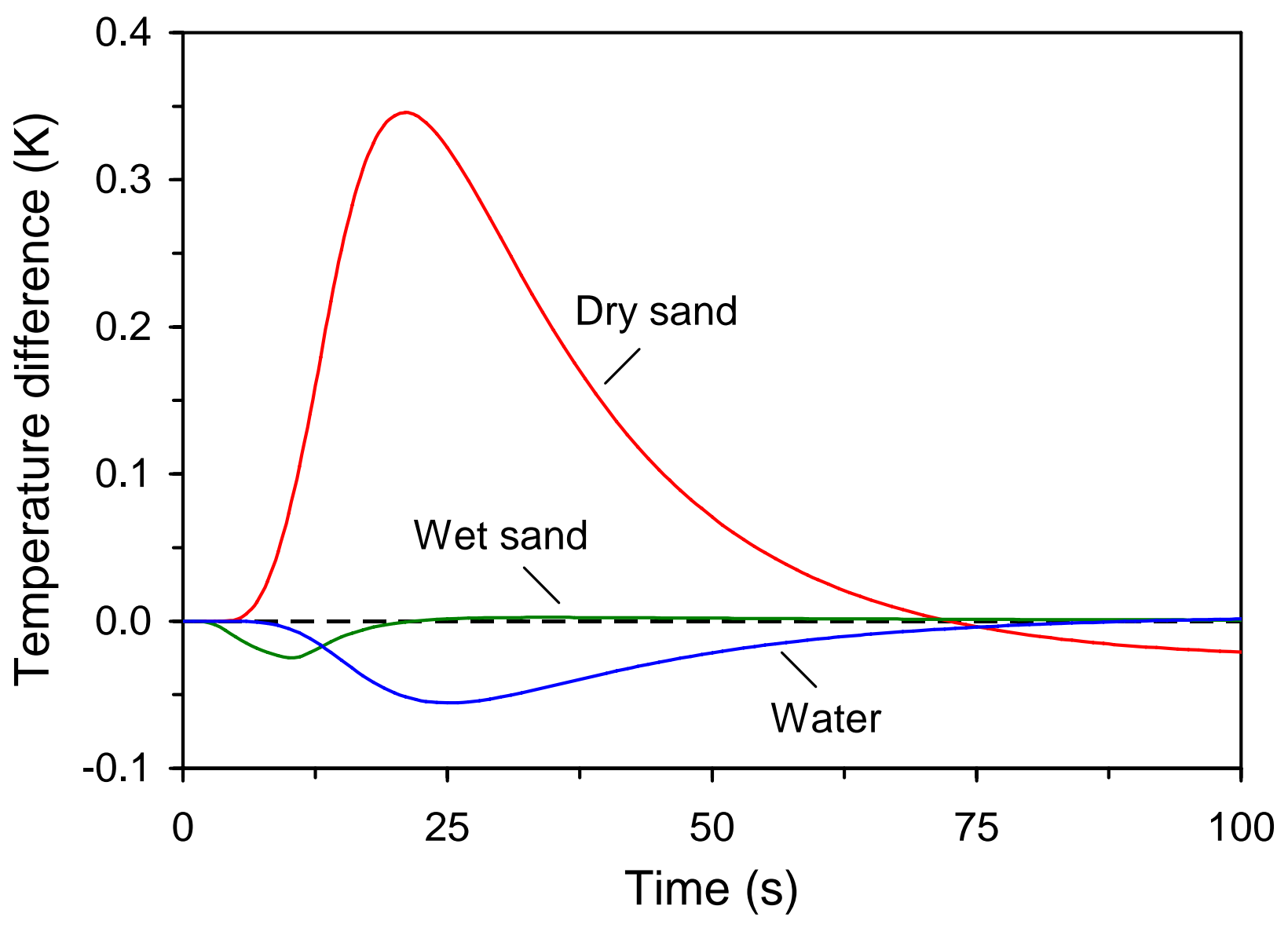


Figure 10

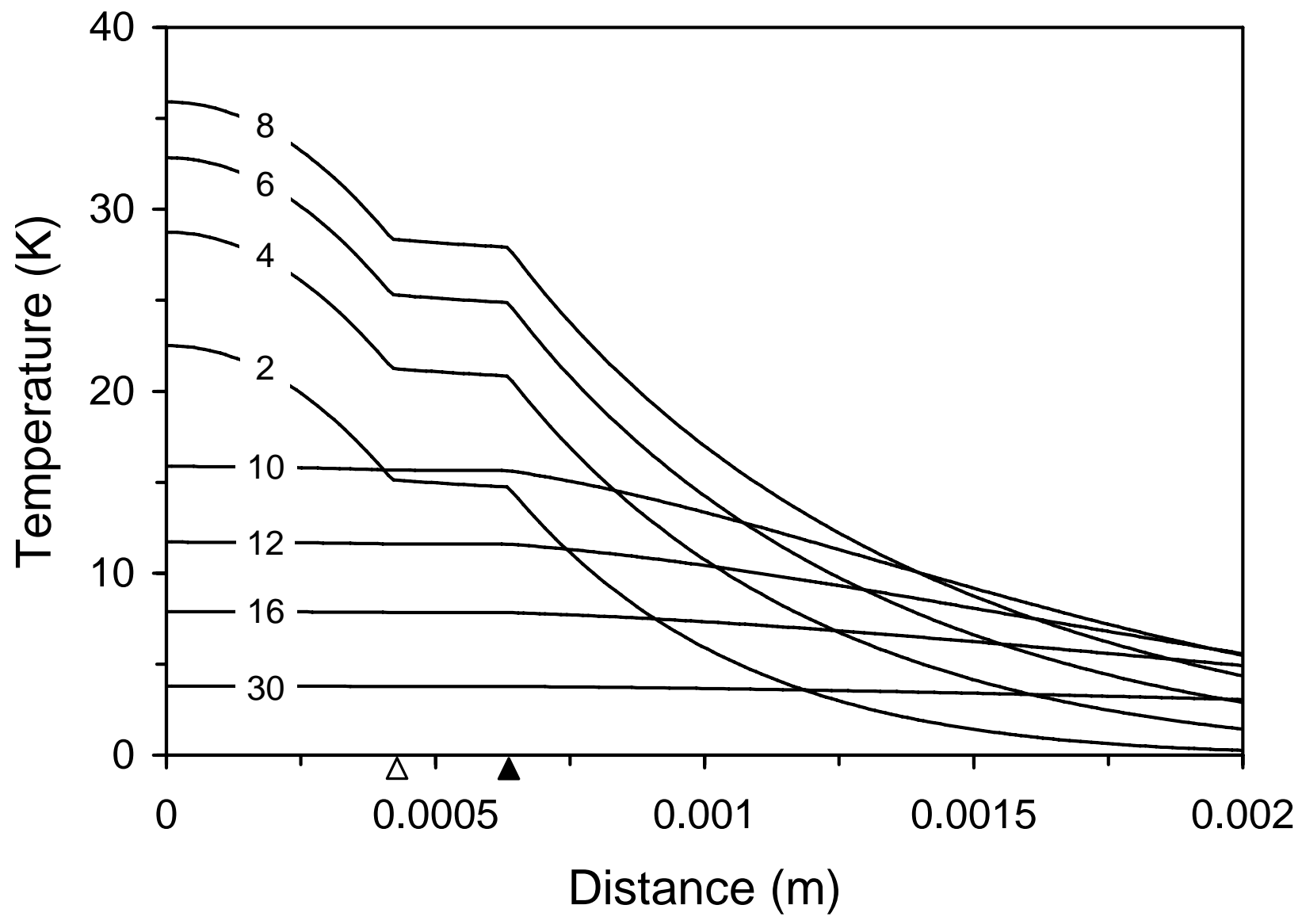

